\title{
Review Article \\ Collectivity in Small Collision Systems: An Initial-State Perspective
}

\author{
Sören Schlichting ${ }^{1,2}$ and Prithwish Tribedy ${ }^{1}$ \\ ${ }^{1}$ Physics Department, Brookhaven National Laboratory, Upton, NY 11973, USA \\ ${ }^{2}$ Department of Physics, University of Washington, Seattle, WA 98195-1560, USA \\ Correspondence should be addressed to Prithwish Tribedy; ptribedy@bnl.gov
}

Received 29 June 2016; Accepted 26 September 2016

Academic Editor: Smarajit Triambak

Copyright (C) 2016 S. Schlichting and P. Tribedy. This is an open access article distributed under the Creative Commons Attribution License, which permits unrestricted use, distribution, and reproduction in any medium, provided the original work is properly cited. The publication of this article was funded by $\mathrm{SCOAP}^{3}$.

\begin{abstract}
Measurements of multiparticle correlations in the collisions of small systems such as $\mathrm{p}+\mathrm{p}, \mathrm{p} / \mathrm{d} /{ }^{3} \mathrm{He}+\mathrm{A}$ show striking similarity to the observations in heavy-ion collisions. A number of observables measured in the high-multiplicity events of these systems resemble features that are attributed to collectivity driven by hydrodynamics. However, alternative explanations based on initialstate dynamics are able to describe many characteristic features of these measurements. In this brief review, we highlight some of the recent developments and outstanding issues in this direction.
\end{abstract}

\section{Introduction}

Relativistic collisions of hadrons and nuclei at the modern colliders provide unique testing ground for $\mathrm{QCD}$ at high energies. Over a decade of experimental measurements for a wide range of energies and collision systems have been dedicated to studying the properties of the matter formed in such collisions. A number of measurements have provided strong indications that the QCD matter formed in the collisions of two nuclei $(\mathrm{A}+\mathrm{A})$ behaves like a strongly interacting fluid that exhibits collectivity [1-4]. Consistent measurements of strong radial and anisotropic flow, jet-quenching, and so forth have convinced us to establish such properties of the medium. Small system collisions have initially been thought of providing benchmarking measurements for the observations in heavy-ion collisions.

Very recently, several striking observations have been made in $\mathrm{p}+\mathrm{p}, \mathrm{p}+\mathrm{A}, \mathrm{d}+\mathrm{A}$, and ${ }^{3} \mathrm{He}+\mathrm{A}$ collisions [5-13]. Observations in high-multiplicity events for such small collision systems seem to resemble features that are common to $\mathrm{A}+\mathrm{A}$ collisions and very often attributed to decisive signatures of collectivity due to hydrodynamic evolution. Such observations include appearance of azimuthal correlations that extend in long range rapidity known as ridge, which is also quantified in terms of Fourier harmonic coefficient of $v_{n}$, strong multiplicity dependence of mean transverse momentum, HBT radii, and many others.

An outstanding question remains whether such systematics have a collective origin that can be attributed to hydrodynamic evolution like in $\mathrm{A}+\mathrm{A}$ collisions or a natural consequence due to initial-state dynamics that appear in the final-state observables or a combination of both. Several alternative approaches based on final-state [14-23] and initialstate [24-40] dynamics have provided competing arguments in this context. Interestingly, such a debate on the relative importance of initial-state [41] and final-state effects [42] also took place in the early 90 s while addressing the experimental data from $\mathrm{p}+\overline{\mathrm{p}}$ collisions at the Tevatron.

In this review, we provide a brief overview on the experimental findings and outline a general theory perspective on the collective phenomena observed in small systems. Subsequently, we focus specifically on theoretical explanations based on the initial-state dynamics. We discuss different theoretical approaches to the calculations and contrast the 
results with experimental findings. We conclude with a brief summary of the present status and perspectives for future studies.

1.1. Experimental Overview. The current debate of collectivity in small systems was triggered by the discovery of ridgelike correlations that extend over a long range in rapidity in high-multiplicity $\mathrm{p}+\mathrm{p}$ collisions by the CMS collaboration [5]. Such long range structure of azimuthal correlations was previously seen in heavy-ion collisions at RHIC [43-45] and LHC [46] and generally attributed to the nearly boost invariance structure of the azimuthal correlations driven by hydrodynamic flow. At the same time, causality arguments suggest [47] that such correlations must develop at the very early stages of the collisions, indicating the strong influence of initial-state dynamics on such observable. While the observation of similar ridge-like structures made in highmultiplicity $\mathrm{p}+\mathrm{Pb}$ collisions at the LHC was expected [6-8], a surprise was that the signal strength at the same multiplicity was stronger in $\mathrm{p}+\mathrm{Pb}$ compared to $\mathrm{p}+\mathrm{p}$. The latest inclusion to these measurements is the highest energy $\mathrm{p}+\mathrm{p}$ collisions so far at $13 \mathrm{TeV}[12,13]$. Including the $13 \mathrm{TeV}$ and $7 \mathrm{TeV}$ data, both ATLAS and CMS collaborations have demonstrated that the strength of ridge correlations (characterized by the nearside yield) at a given multiplicity is independent of collision energy. However, a more pronounced energy dependence appears to be present in higher-order cumulants such as $c_{2}\{4\}=-v_{2}^{4}\{4\}$ which unlike the case at lower energies appears to exhibit a clear sign change as a function of multiplicity in $13 \mathrm{TeV} p+p$ collisions [48]. Meanwhile, RHIC, being a versatile machine, has collided ${ }^{3} \mathrm{He}+\mathrm{Au}$ [11] along with $\mathrm{d}+\mathrm{Au}$ $[9,10]$ and $\mathrm{p}+\mathrm{Au}[49]$ to perform similar measurements of azimuthal correlations. Such measurements indicate the presence of significant $v_{2}$ and $v_{3}$ in $\mathrm{p}+\mathrm{Au}, \mathrm{d}+\mathrm{Au}$, and ${ }^{3} \mathrm{He}+\mathrm{Au}$, the systematics of which is very similar to what is commonly seen in $\mathrm{A}+\mathrm{A}$ collisions. Analyses common to $\mathrm{A}+\mathrm{A}$ have been repeated in both $\mathrm{p}+\mathrm{Pb}$ and $\mathrm{p}+\mathrm{p}$ at $\mathrm{LHC}$ by triggering on high-multiplicity events. Measurements of higher-order harmonics of azimuthal anisotropy $v_{n}$, mass dependence of $v_{n}$, higher-order cumulants of $v_{2}\{n\}, n \geq 4$, and many others are now available (for a comprehensive review, we refer the reader to $[50,51])$.

By now, a number of intriguing results observed in $\mathrm{p}+\mathrm{p}$ and $\mathrm{p} / \mathrm{d} /{ }^{3} \mathrm{He}+\mathrm{A}$ have accumulated, including a strong multiplicity dependence of average transverse momentum $\left\langle p_{T}\right\rangle$ that is attributed to radial flow as well as the observation of sizable Fourier harmonic coefficients $v_{n}\left(p_{T}\right)$ up to $n=4$ and its higher-order moments of the azimuthal correlation generally attributed to anisotropic flow. Most importantly, several characteristics, such as the mass dependence of both $\left\langle p_{T}\right\rangle$ and $v_{n}\left(p_{T}\right)$, have been found to be similar to what is seen in $\mathrm{A}+\mathrm{A}$ collisions.

However, it is worth mentioning that some striking contrasts also exist. Unlike in A+A collisions, where the observation of jet-quenching has been one of the pillars of the discovery of a strongly interacting Quark-Gluon plasma (QGP) [52, 53], so far no evidence of (mini) jetquenching has been found in small systems [54-57]. Even though the standard jet-quenching analysis in small systems is complicated due to trigger bias effects, the absence of such phenomena may provide important insights with regard to the theoretical interpretation of the observed phenomena.

1.2. General Theoretical Perspectives. It is useful to first address the question about the origin of long range azimuthal correlations (shown in Figure 1) from a more general point of view and formulate our theoretical expectations based on previous observations in small and large systems. While causality arguments imply that any long range rapidity correlation must originate from the very early stages of the collision [47], this leaves open the question how the observed momentum space correlations are created dynamically during the spacetime evolution. Specifically, one can, at least from a theoretical point of view, distinguish two different mechanisms whereby momentum space correlations of hadrons produced in the final-state reflect

(i) intrinsic momentum space correlations of the partons produced in initial (semi-) hard scatterings

(ii) position space correlations between initial-state partons, for example, the initial-state geometry, which are transformed into momentum space correlations due to final-state interactions.

While, in any realistic scenario, both kinds of correlations (i) and (ii) contribute to the long range azimuthal correlations, their relative strength depends on the magnitude of final-state effects. In low-multiplicity $\mathrm{p}+\mathrm{p}$ collisions, for example, the dominant source of long range azimuthal correlations is due to the production of back-to-back (mini-) jets. Since in this case the density of produced partons is low, the typical (semi-) hard partons produced in the initial scattering escape the interaction region without final-state effects significantly affecting their back-to-back correlation. Considering on the other hand soft particle production amidst large parton densities in nucleus-nucleus collisions, it is well established that the azimuthal anisotropy of, for example, $p_{T} \lesssim 1 \mathrm{GeV}$ particles is dominated by the final-state response to the initial-state geometry. In this case, the meanfree path of a typical (semi-) hard parton is small compared to the system size, such that the initial-state momentum correlations of $\sim \mathrm{GeV}$ partons are destroyed during the equilibration process. Therefore, the subsequent dynamics of the equilibrated QGP can be accurately described by relativistic hydrodynamics.

Even though it is sometimes possible to choose the kinematics such that one mechanism dominates over the other, there are various examples in between where both initialstate and final-state effects are important. One prominent example includes the behavior of jets in heavy-ion collisions. While highly energetic jets can escape the interaction region without equilibrating, they can loose a significant part of their energy through interactions with the softer medium. Even though the dominant correlation of the leading high$p_{T}$ particles is still due to the initial back-to-back correlation, the path length dependence of the energy loss in the medium also leads to an additional correlation with the initial-state 

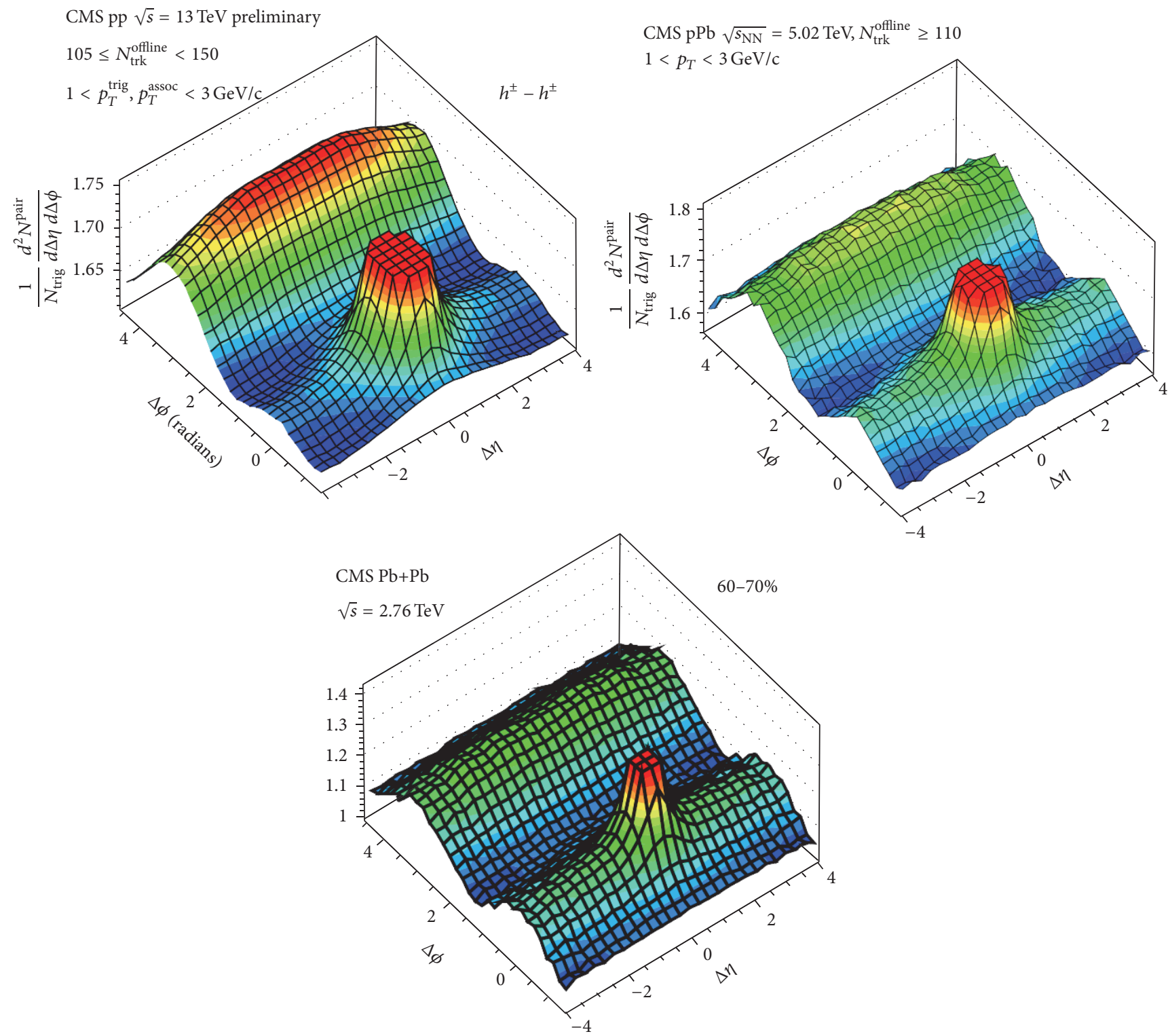

FIGURE 1: Two-particle correlation function in relative pseudorapidity and azimuthal angle showing long range ridge-like structure in highmultiplicity $\mathrm{p}+\mathrm{p}, \mathrm{p}+\mathrm{Pb}$ as compared to peripheral $\mathrm{Pb}+\mathrm{Pb}$ collisions. Figures are taken from $[6,13,46]$.

geometry. Such correlations are reflected, for example, by the high-momentum $v_{n}\left(p_{T}\right)$ measuring correlations between soft and hard particles.

Clearly, the aforementioned examples illustrate that it is important to consider both initial-state momentum space correlations and the response to the initial-state geometry due to final-state effects in order to describe azimuthal correlations in small systems over a wide kinematic range. Our qualitative expectation is illustrated in Figure 2, where the azimuthal correlation strength due to initial-state and finalstate effects is shown versus the event multiplicity, for example, in $\mathrm{p}+\mathrm{p}$ collisions for a fixed transverse momentum range, for example, 1-3 GeV. Based on our discussion, we expect that in low-multiplicity or minbias events the azimuthal correlations between $1-3 \mathrm{GeV}$ particles are predominantly due to back-to-back minijets (peaked at $\Delta \phi=\pi$ ). With increasing event multiplicity, the contribution from multiparton processes, such as the "Glasma graphs" (Section 2.3.3), becomes increasingly important resulting in azimuthal correlations that have a symmetric structure in relative azimuthal angle $\Delta \phi$ around $\pi / 2$. When increasing the multiplicities even further, final-state interactions in this transverse momentum region can no longer be neglected at some point and lead to a depletion of initial-state correlations. Even though minijets do not fully equilibrate yet, the system starts to show a response to the initial-state geometry, which in this low opacity region is presumably dominated by the path length dependence of the parton energy loss, also referred to as parton escape mechanism [58]. Ultimately, in the limit of very high multiplicities, minijets are fully quenched, resulting in 


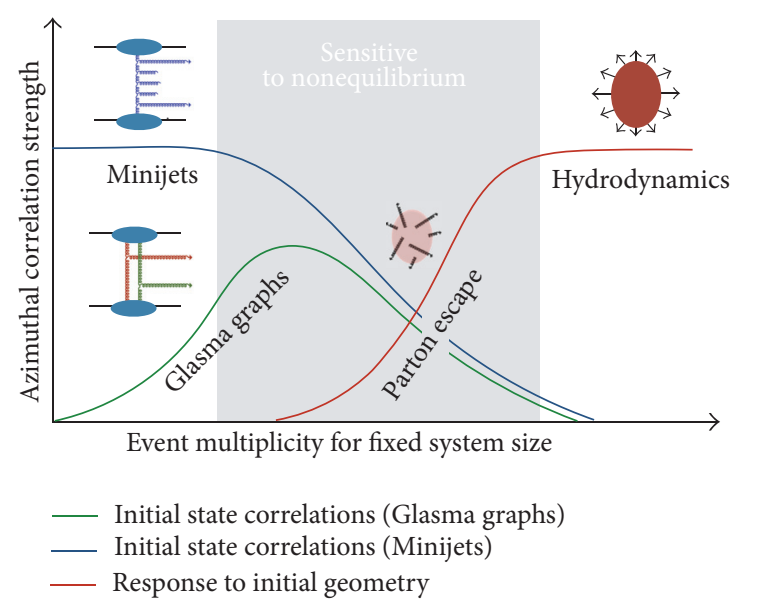

FIGURE 2: Illustration of long range azimuthal correlations in small systems, a slightly modified version of the figure from [59].

the formation of a thermalized medium and the complete loss of initial-state momentum space correlations. In this high opacity regime, azimuthal correlations are dominated by the response to initial geometry described by a hydrodynamic expansion of a thermalized Quark-Gluon plasma.

One can attempt to further estimate the multiplicities corresponding to the transitions from the initial-state to the final-state dominated regime, exploiting recent theoretical progress in the understanding of the equilibration process [65]. Since the equilibration time at weak coupling corresponds to the time scale when a semihard parton $\sim Q_{s}$ looses all its energy to form a soft thermal bath, one naturally expects the crossover from the initial-state to final-state dominated regime to occur when the associated equilibration time $\tau_{\text {eq }}$ becomes comparable to the system size $R$. Conversely, as long as $\tau_{\text {eq }} \gg R$ typical semihard partons escape without encountering significant final-state interactions, whereas for $\tau_{\text {eq }} \ll R$ semihard partons are fully quenched, equilibrium is reached early on and the dynamics is dominated by the subsequent hydrodynamic expansion. Based on the estimate of the equilibration time $Q_{s} \tau_{\text {eq }} \simeq 10(\eta / s)_{T_{\text {eq }}}^{4 / 3}\left(g^{2} N_{c}\right)^{1 / 3} \simeq 10$ for $(\eta / s)_{T_{\mathrm{eq}}} \simeq 5 / 4 \pi$ at realistic coupling $g^{2} N_{c} \simeq 10[66,67]$ and the multiplicity $d N / d y \simeq \xi Q_{s}^{2} \pi R^{2}$ with $\xi \simeq 1 / 4$ [68], we obtain that

$$
\frac{\tau_{\mathrm{eq}}}{R} \simeq \sqrt{\frac{100}{d N / d y}}
$$

corresponding to a crossover at around $d N / d y \sim 100$, which in fact is much larger than the minbias multiplicities reached in $\mathrm{p}+\mathrm{p}$ or $\mathrm{p}+\mathrm{Pb}$ collisions (the typical values of $d N / d y$ in minbias $\mathrm{p}+\mathrm{p}$ and $\mathrm{p}+\mathrm{Pb}$ collisions at $\mathrm{LHC}$ are about $\sim 6$ and $\sim 17$ [69] and [70], resp.). We caution however that the estimate in (1) is inferred from leading order weak-coupling calculations and should only serve as a ballpark figure.

Beyond simple analytic estimates, probably a promising alternative approach is to directly attempt an extraction of the boundaries between the different regimes through detailed comparisons of theory and experiment. While a firstprinciple theoretical description is complicated throughout most of the multiplicity regimes shown in Figure 2, significant theoretical progress has been made in understanding the features of initial-state correlations in the regime where finalstate effects can be neglected. In the following, we will review the theoretical computation of initial-state correlations in the color glass condensate (CGC) effective field theory of highenergy QCD and critically assess to what extent these calculations are compatible with the experimental observations.

\section{Multiparticle Production in the CGC Framework}

2.1. High-Multiplicity Events. Experimental observations suggest that long range ridge-like correlations in small colliding systems appear in high-multiplicity events. Before we turn to a more detailed discussion of possible mechanisms to produce such correlations, a first necessary step is to understand the origin of high-multiplicity events that populate the long tail of experimental multiplicity distributions. Considering the most elementary case of $p+p$ collisions, high-multiplicity events are a consequence of three major sources of fluctuations:

(1) Geometry of collisions

(2) Intrinsic saturation scale of the proton

(3) Distribution of color charge density inside the protons.

While one naturally expects a strong impact parameter dependence of the multiplicity in $\mathrm{p}+\mathrm{p}$ collisions [71], the importance of additional sources of initial-state fluctuations have only been realized recently. Significant progress in including all of the above into a consistent phenomenological description has been made within the IP-Glasma model which is based on the framework of CGC $[72,73]$.

Nonperturbative large $x$ effects, which are not captured in the conventional CGC framework, are expected to give rise to fluctuations of the intrinsic saturation scale of the proton [74-79] as illustrated in Figure 3(b). In [61], intrinsic fluctuations of the proton saturation scale were introduced in the IP-Glasma model [61] according to a distribution:

$$
P\left(\ln \left(\frac{Q_{s}^{2}}{\left\langle Q_{s}^{2}\right\rangle}\right)\right)=\frac{1}{\sqrt{2 \pi \sigma}} \exp \left(-\frac{\ln ^{2}\left(Q_{s}^{2} /\left\langle Q_{s}^{2}\right\rangle\right)}{2 \sigma^{2}}\right) \text {. }
$$

with the variance $\sigma^{2}$ adjusted previously to the inclusive charged particle multiplicity and rapidity distributions in $\mathrm{p}+\mathrm{p}$ collisions over a wide range of energies $0.2-7 \mathrm{TeV}$ [61]. An interesting consequence of such fluctuations is that, even in symmetric collision systems such as $\mathrm{p}+\mathrm{p}$, event-by-event fluctuations of the saturation scale of each proton lead to an asymmetry of the rapidity distribution of the produced particles on an event-by-event basis [80]. Consequently, highmultiplicity $\mathrm{p}+\mathrm{p}$ collisions in particular are always asymmetric and in a sense expected to look more like $\mathrm{p}+\mathrm{A}$ collisions.

The saturation scale and the geometric profile of the proton, which are the two most important ingredients in the 

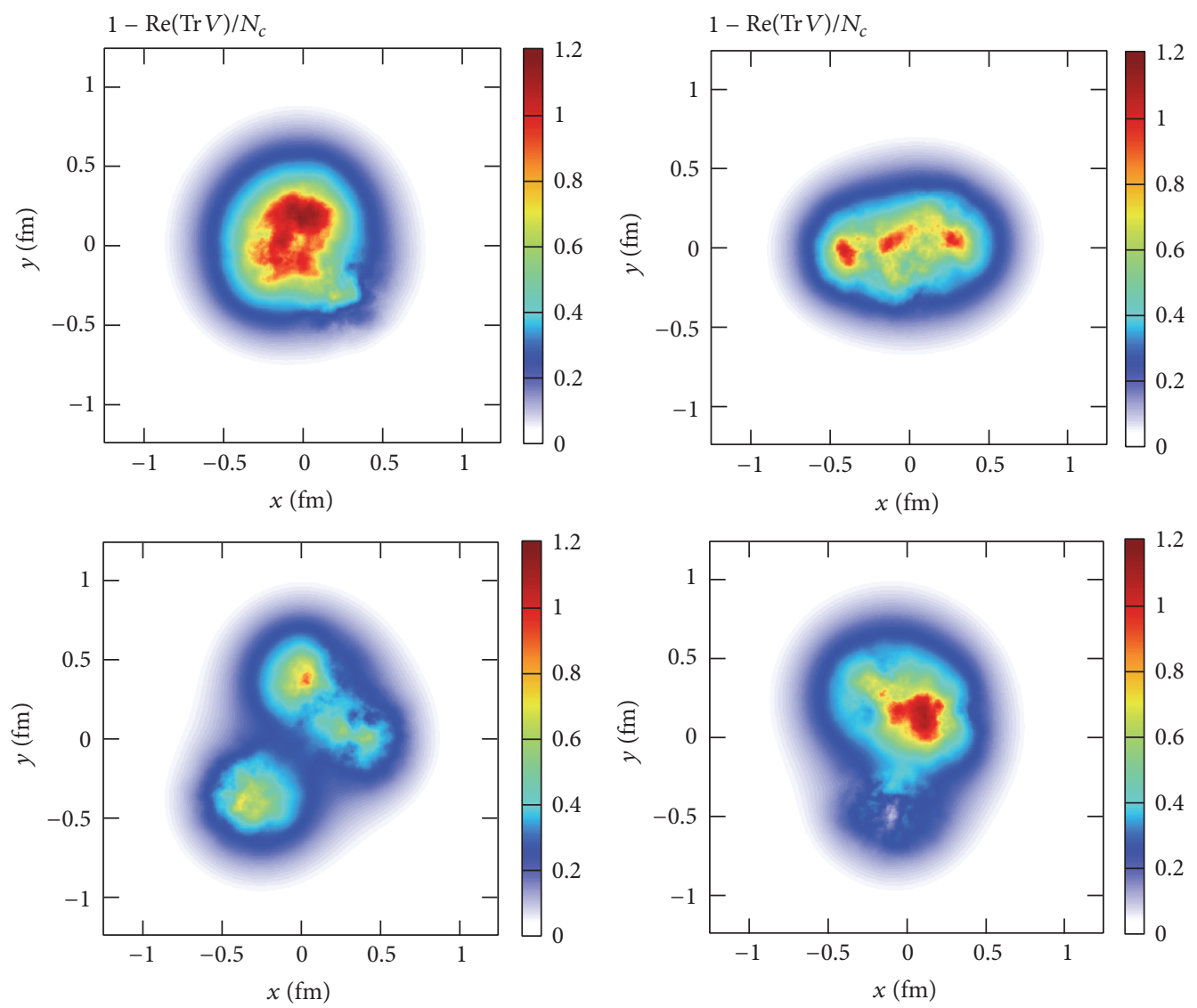

(a)

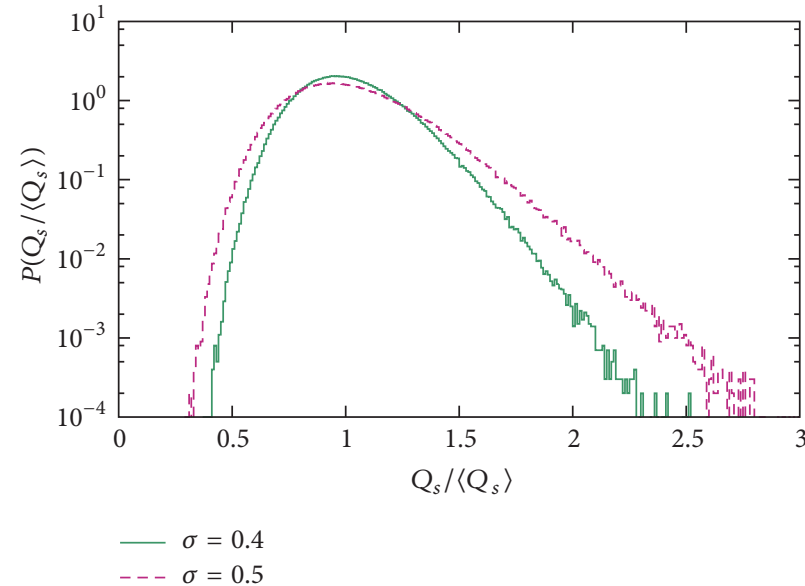

(b)

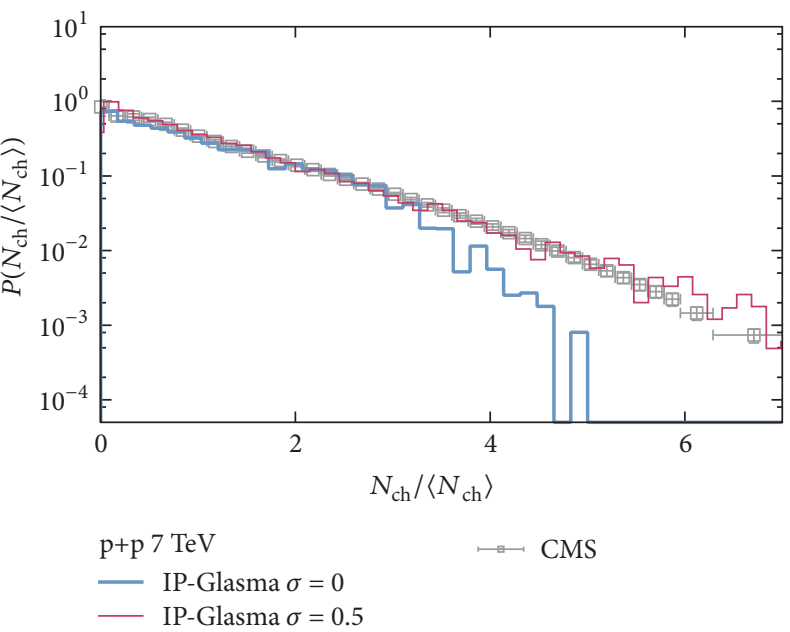

(c)

FIGURE 3: (a) Distribution of gluon density around valance quarks inside a proton represented by the real part of Wilson lines from [60]. (b) Distribution of the saturation scale of a proton driven by stochastic dipole splitting. (c) Effect of intrinsic fluctuation of proton saturation scale on the probability distribution of multiplicity $\left(\sigma=0\right.$ corresponds to no fluctuation of $\left.Q_{s}\right)$. Figures (b) and (c) are from [61].

IP-Glasma model, are obtained from the IP-Sat parameterization of the HERA DIS data [81,82]. While in the original IP-Sat model the proton shape was assumed to be round, recent modification to IP-Sat has been done by assuming the gluon density to be distributed around three valence quarks inside the proton (see Figure 3(a)) $[60,83]$ and several other models have started to include similar kinds of fluctuations $[84,85]$. Interestingly, such subnucleon scale fluctuations can be constrained by incoherent diffractive vector meson production and improved agreement with existing HERA data can be achieved [60]. It has also been pointed out that geometric fluctuations of the proton can provide a dynamical 


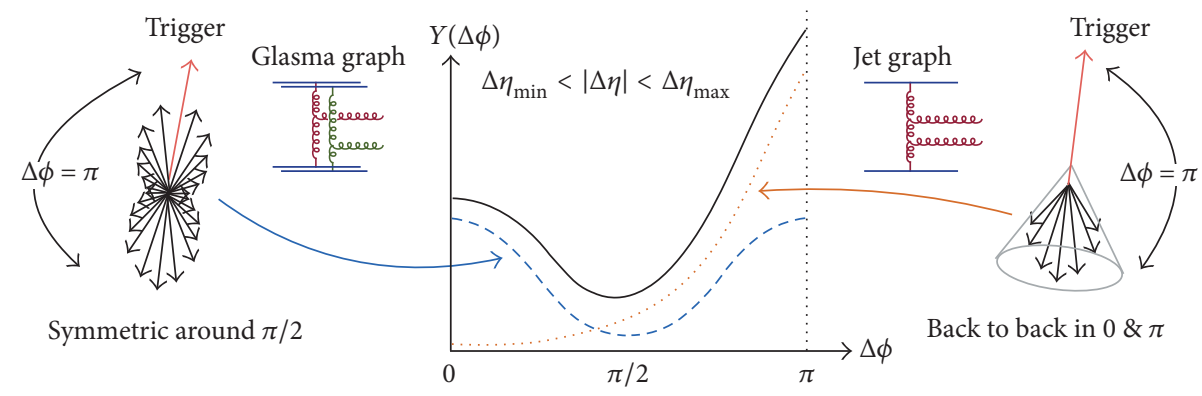

FIGURE 4: A cartoon showing the contributions of dijet and Glasma graphs in two-particle correlation function $Y(\Delta \phi)$ integrated over a broad range of $|\Delta \eta|$. This is a slightly modified version of the figure from [62].

explanation for the "hollowness effect" observed in elastic $\mathrm{p}+\mathrm{p}$ scattering at LHC energies [86] and it would further be interesting to explore to what extent the modeling of the proton geometry can be aided by first-principle lattice QCD calculations.

With the initial-state including different sources of fluctuations constrained by the HERA data, the $n$-particle production probability $P(n)$ can be computed within the color glass condensate framework. For a given configuration of initial color charge, the $n$-particle distribution is a negative binomial distribution (NBD) with mean and width related to the saturation scale [87]. Due to fluctuation of impact parameter, a convolution of many such NBDs gives rise to the final probability distribution of multiplicity. However, it has been demonstrated that such distribution is narrower compared to the data. Only after including the intrinsic fluctuations of the proton saturation scale, one can describe the tails of the experimental multiplicity distributions (Figure 3(c)). Since multiplicity is dominated by low momentum $\left(p_{T}<\right.$ $1 \mathrm{GeV}$ ) gluons, it is very challenging to implement a scheme of fragmentation for the production of soft hadrons. Therefore, the number of produced charged particles is generally taken to be proportional to the number of gluons. The results shown in Figure 3 indicate that the high-multiplicity events that populate the tail of $P(n)$ distributions are generated due to rare high color charge density configurations of the wave functions of the colliding systems. In the next section, we argue that the same underlying dynamics that leads to the origin of high-multiplicity events also drives the systematics of multiparticle correlations in small collision systems.

2.2. Qualitative Discussion of Initial-State Correlations. Multiparticle production in Quantum Chromo Dynamics (QCD) naturally leads to correlations between particles produced in high-energy collisions. A complete theoretical understanding of these effects though is extremely challenging. Nevertheless, significant progress has been achieved in recent years based on the CGC effective field theory (EFT) of high-energy QCD, which provides the basis for phenomenological applications at RHIC and LHC energies.

Let us focus our discussion on the origin and systematics of the two-particle correlations seen at LHC. By far, the most well established source of long range two-particle azimuthal correlations is due to the production of back-to-back dijets.
Such processes (also referred to as "Mueller-Navelet" jets [88]) are depicted in the right panel of Figure 4 and can be computed within standard perturbative QCD. Dijet production is kinematically constrained to produce only away-side (peaked at $\Delta \phi=\pi$ ) collimations and dominates in lowmultiplicity or minbias events. However, in high-multiplicity events, one is probing rare configurations of the proton where in addition to the production of dijets from a single hard scattering, multiparton processes become increasingly important. A first calculation of these effects in the CGC framework was based on evaluating the associated Feynman diagrams referred to as "Glasma graphs," depicted in the left panel of Figure 4. Such graphs give rise to nonfactorizable two-particle correlations that have a symmetric structure in relative azimuthal angle $\Delta \phi$ around $\pi / 2$ (see Figure 4). When decomposed in terms of the Fourier coefficients of the particle distributions, they give rise to nonzero even harmonics $v_{n}$. Beyond the lowest order processes depicted in the left panel of Figure 4, further contributions to the azimuthal collimations come from the multiple scattering of partons leading to both even and odd $v_{n}$. Such processes can be included in a classical Yang-Mills description and will be discussed in more detail in a following section.

Since interference effects between Glasma graphs and jet graphs vanish to lowest order in the kinematic regime $\Lambda_{\mathrm{QCD}} \ll Q_{s} \lesssim p_{T}, q_{T}$, the resulting two-particle correlations function as a direct sum of both contributions:

$$
\begin{aligned}
\frac{d^{2} N^{\text {corr. }}}{d^{2} \mathbf{p}_{T} d^{2} \mathbf{q}_{T} d y_{p} d y_{q}}= & \frac{d^{2} N_{\mathrm{Glasma}}^{\text {corr. }}}{d^{2} \mathbf{p}_{T} d^{2} \mathbf{q}_{T} d y_{p} d y_{q}} \\
& +\frac{d^{2} N_{\text {Jet }}^{\text {corr. }}}{d^{2} \mathbf{p}_{T} d^{2} \mathbf{q}_{T} d y_{p} d y_{q}} .
\end{aligned}
$$

The relative strength of the dijet production represented by the "jet graph" and the "Glasma graphs" determines the features of the observed dihadron correlations as shown in Figure 4. In high-multiplicity events, the Glasma graphs are enhanced by a relative factor of $\alpha_{s}^{-4}$ compared to the "jet graphs"; one therefore naturally expects to see a pronounced near-side collimation at $\Delta \phi \sim 0$ that extends over a wide range of rapidity referred to as the "near-side ridge." The fact that near-side collimation extends far in rapidity is a consequence of the nearly boost invariant nature of the 
Glasma gluon fields. While qualitatively these features are indeed present in the experimental data, of course it requires detailed theory calculations to establish the quality of agreement. In the remainder of this section, we will outline the essential steps in the computation of initial-state correlations in the CGC framework. A summary of comparisons with experimental results is presented in Section 3.

In the CGC framework, colliding protons and nuclei are effectively described as static sources of color charge on the light-cone that generate color currents:

$$
J^{\nu}=\delta^{\nu \pm} \rho_{A(B)}\left(x^{\mp}, \mathbf{x}_{\perp}\right) .
$$

The color charge densities $\rho_{A(B)}\left(x^{ \pm}, \mathbf{x}_{\perp}\right)$ in each colliding hadron or nucleus fluctuate from event to event and their statistical properties are constrained by independent measurements. Computation of multiparticle production in the CGC framework is based on the calculation of the classical Yang-Mills fields created from such color currents by solving the Yang-Mills equations:

$$
\left[D_{\mu}, F^{\mu \nu}\right]=J^{\nu}
$$

Different theoretical descriptions within the CGC framework employ different levels of approximation which are discussed in more detail in the following.

2.3. Perturbative Computation. In the perturbative framework, one tries to obtain an analytical solution of the gauge fields by performing order-by-order expansion of (5) in powers of the color sources $\rho_{A(B)}$. In the dilute-dense framework which assumes lowest order in $\rho_{A}$ and all orders in $\rho_{B}$ or in the dilute-dilute framework, one can derive analytical expressions for $\mathrm{n}$-gluon production in the $k_{\perp}$-factorized form [89]. The essential ingredients to such factorization relations are the correlator of the dilute-sources in $\rho_{A}$ or the Wilson lines corresponding to the dense source $\rho_{B}$ in momentum space. Such correlators are represented as unintegrated gluon distributions (UGDs) $\Phi\left(x, k_{\perp}\right)$ and can be expressed in terms of $\mathscr{T}\left(x, r_{\perp}\right)$, the forward scattering amplitude of a quarkantiquark dipole of transverse size $r_{\perp}$ on a proton/nuclear target, through the expression

$$
\begin{aligned}
& \Phi\left(x, k_{\perp}\right) \\
& =\frac{\pi N_{c} k_{\perp}^{2}}{2 \alpha_{s}} \int_{0}^{\infty} d r_{\perp} r_{\perp} J_{0}\left(k_{\perp} r_{\perp}\right)\left[1-\mathscr{T}\left(x, r_{\perp}\right)\right]^{2} .
\end{aligned}
$$

The $x$-dependence of $\Phi$ is determined by the rapidity $(Y=\ln (1 / x))$ evolution of the dipole scattering amplitude implemented in the Balitsky-Kovchegov (BK) renormalization equation $[90,91]$ which is a simplified form of the JIMWLK renormalization equations [90, 92-95]. The leading order expression of the $\mathrm{BK}$ equation is given by

$$
\begin{aligned}
& \frac{\partial T(\mathbf{r}, Y)}{\partial Y}=\int_{\mathbf{r}_{1}} \mathscr{K}\left(\mathbf{r}, \mathbf{r}_{1}\right)\left[T\left(\mathbf{r}_{1}, Y\right)+T\left(\mathbf{r}_{2}, Y\right)\right. \\
& \left.\quad-T(\mathbf{r}, Y)-T\left(\mathbf{r}_{1}, Y\right) T\left(\mathbf{r}_{2}, Y\right)\right]
\end{aligned}
$$

where $\mathbf{r}_{2} \equiv \mathbf{r}-\mathbf{r}_{1}$ and $\mathscr{K}$ is the BFKL kernel. The implementation of the kernel $\mathscr{K}$ often used for phenomenology includes a running coupling next-to-leading-log (NLL) correction to $\mathrm{BK}$ and is referred to as the $\mathrm{rcBK}$ equation [96] given by

$$
\begin{aligned}
& \mathscr{K}\left(\mathbf{r}, \mathbf{r}_{1}, \mathbf{r}_{2}\right)=\frac{\alpha_{s}(\mathbf{r}) N_{c}}{\pi}\left[\frac{\mathbf{r}^{2}}{\mathbf{r}_{1}^{2} \mathbf{r}_{2}^{2}}+\frac{1}{\mathbf{r}_{1}^{2}}\left(\frac{\alpha_{s}\left(\mathbf{r}_{1}^{2}\right)}{\alpha_{s}\left(\mathbf{r}_{2}^{2}\right)}-1\right)\right. \\
& \left.+\frac{1}{\mathbf{r}_{2}^{2}}\left(\frac{\alpha_{s}\left(\mathbf{r}_{2}^{2}\right)}{\alpha_{s}\left(\mathbf{r}_{1}^{2}\right)}-1\right)\right] .
\end{aligned}
$$

Equation (8) requires an initial condition for $\mathscr{T}_{A, B}(x=$ $\left.x_{0}, r_{\perp}\right)$, one choice of which is the McLerran-Venugopalan (MV) model $[97,98]$ with a finite anomalous dimension $\gamma$ given by

$$
\begin{aligned}
\mathscr{T} & \left(x_{0}, r_{\perp}\right) \\
& =1-\exp \left[-\left(\frac{r_{\perp}^{2} Q_{s 0}^{2}}{4}\right)^{\gamma} \ln \left(\frac{1}{r_{\perp} \Lambda_{\mathrm{QCD}}}+e\right)\right],
\end{aligned}
$$

where $Q_{s 0}^{2}$ is a nonperturbative scale. This MV-like parameterization is constrained by global fits to the DIS data [99] although it must be noted that MV along with BK evolution does not include spatial geometric structure of the proton. The scattering amplitude is known to have a strong dependence on the impact parameter [81] which is incorporated in other parameterizations such as IP-Sat [82, 100] or b-CGC [81] models of DIS. More recently, significant progress consistently including the full NLL corrections to BK evolution into DIS fits [101-103] has been made. However, so far, this has not been included in phenomenological studies of $\mathrm{p}+\mathrm{p}$ and $\mathrm{p}+\mathrm{A}$ collisions

With the unintegrated gluon distribution obtained from (6), one can estimate the production of $n$-gluons using the $k_{\perp}$ factorization approach. In the following section, we describe the approach for single-inclusive, double-inclusive, and jet production essential for the phenomenology in $\mathrm{p}+\mathrm{p}$ and $\mathrm{p}+\mathrm{A}$ collisions.

2.3.1. Single-Inclusive Gluon Distribution. The single-inclusive gluon production corresponds to the simplest process that describes the emission of a single gluon of momentum $\mathbf{p}_{T}$ which in the perturbative framework can be written in the $k_{\perp}$-factorized form as

$$
\frac{d N}{d y_{p} d^{2} \mathbf{p}_{T}}=\frac{8 \alpha_{s}}{(2 \pi)^{6} C_{F}} \frac{S_{\perp}}{\mathbf{p}_{T}^{2}} \int_{\mathbf{k}_{T}} \Phi_{A}\left(\mathbf{k}_{T}\right) \Phi_{B}\left(\mathbf{p}_{T}-\mathbf{k}_{T}\right),
$$

where $C_{F}=\left(N_{c}^{2}-1\right) / 2 N_{c}$ and $S_{\perp}$ is the transverse overlap area. It must be noted that this $k_{\perp}$-factorized form of single gluon production has a logarithmic infrared divergence which is generally regulated by putting a lower $p_{T, \text { min }}$ cut. It must be noted that (10) assumes that the dependence on transverse geometry or the impact parameter of collisions has already been integrated out and absorbed into $S_{\perp}$; a more general expression in such a context can be found in [89]. 


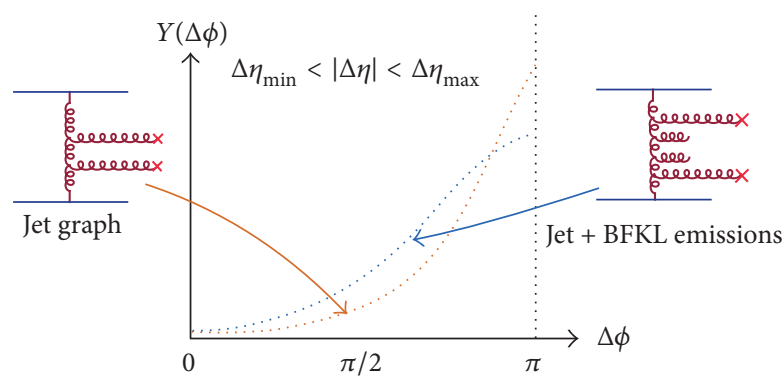

Figure 5: A cartoon showing the contributions of dijet and Glasma graphs in two-particle correlation function $Y(\Delta \phi)$ integrated over a broad range of $|\Delta \eta|$.

2.3.2. Dijets. In the perturbative framework, estimation of the two-particle correlations in $\mathrm{p}+\mathrm{p}$ and $\mathrm{p}+\mathrm{A}$ collisions is based on a direct computation of dijet and the Glasma graphs in the $k_{\perp}$-factorization approximation [27-29, 31, 40]. Such approximations are valid for momenta above the saturation scale $Q_{s}$ and do not include multiple-scattering effects.

The dijet contribution in this framework is estimated [88, $104,105]$ to be

$$
\begin{gathered}
\frac{d^{2} N_{\mathrm{BFKL}}^{\text {corr. }}}{d^{2} \mathbf{p}_{T} d^{2} \mathbf{q}_{T} d y_{p} d y_{q}}=\frac{32 N_{c} \alpha_{s}^{2}}{(2 \pi)^{8} C_{F}} \frac{S_{\perp}}{\mathbf{p}_{T}^{2} \mathbf{q}_{T}^{2}} \\
\cdot \int_{\mathbf{k}_{0 \perp}} \int_{\mathbf{k}_{3 \perp}} \Phi_{A}\left(\mathbf{k}_{0 \perp}\right) \Phi_{B}\left(\mathbf{k}_{3 \perp}\right) \\
\cdot \mathscr{G}\left(\mathbf{k}_{0 \perp}-\mathbf{p}_{T}, \mathbf{k}_{3 \perp}+\mathbf{q}_{T}, y_{p}-y_{q}\right),
\end{gathered}
$$

where $\mathscr{G}$ is BFKL Green's function that generates gluon emissions between the gluons that fragment into triggered hadrons. The form of $\mathscr{G}$ is given by [31].

$$
\begin{aligned}
\mathscr{G}\left(\mathbf{q}_{a \perp}, \mathbf{q}_{b \perp}, \Delta y\right)= & \frac{1}{(2 \pi)^{2}} \frac{1}{\left(\mathbf{q}_{a \perp}^{2} \mathbf{q}_{b \perp}^{2}\right)^{1 / 2}} \sum_{n} e^{i n \bar{\phi}} \\
& \cdot \int_{-\infty}^{+\infty} d v e^{\omega(\nu, n) \Delta y} e^{i v \ln \left(\mathbf{q}_{a \perp}^{2} / \mathbf{q}_{b \perp}^{2}\right)} .
\end{aligned}
$$

Here, $\omega(\nu, n)=-2 \bar{\alpha}_{s} \operatorname{Re}[\Psi((|n|+1) / 2+i v)-\Psi(1)]$ is the BFKL eigenvalue with $\Psi(z)=d \ln \Gamma(z) / d z$ being the logarithmic derivative of the Gamma function with the effective coupling factor $\bar{\alpha}_{s} \equiv N_{c} \alpha_{s}\left(\sqrt{\mathbf{q}_{a \perp} \mathbf{q}_{b \perp}}\right) / \pi$ and $\bar{\phi} \equiv$ $\arccos \left(\left(\mathbf{q}_{a \perp} \cdot \mathbf{q}_{b \perp}\right) /\left|\mathbf{q}_{a \perp} \| \mathbf{q}_{b \perp}\right|\right)$. For a pair of hadrons with a rapidity separation of $\Delta y \geq 1 / \alpha_{s}, \mathscr{G}$ does the necessary resummation of the rapidity ordered multigluon emissions. The effect of such gluon emission leads to angular decorrelation that affects the observed dihadron correlation [88]. The diagrams corresponding to such BFKL emission and its impact on broadening the away-side structure of the azimuthal dihadron correlation are shown in Figure 5. In the $\alpha_{s} \Delta y \rightarrow 0$ limit, one can obtain the well known form of the dijet cross section expression in the Multi-Regge kinematics (MRK) $[105,106]$ :

$$
\begin{aligned}
& \left.\frac{d^{2} N_{A B}}{d^{2} \mathbf{p}_{T} d^{2} \mathbf{q}_{T} d y_{p} d y_{q}}\right|_{\mathrm{MRK}}=\frac{16 N_{c} \alpha_{s}\left(\mathbf{p}_{T}\right) \alpha_{s}\left(\mathbf{q}_{T}\right)}{(2 \pi)^{8} C_{F}} \frac{S_{\perp}}{\mathbf{p}_{T}^{2} \mathbf{q}_{T}^{2}} \\
& \cdot \int_{\mathbf{k}_{\perp \perp}} \Phi_{A}\left(x_{1}, \mathbf{k}_{1 \perp}\right) \Phi_{B}\left(x_{2}, \mathbf{k}_{2 \perp}\right) .
\end{aligned}
$$

As we discuss in the following section, the quantitative estimation of the dijet cross section is essential for the description of the dihadron correlation observed in highmultiplicity events of $\mathrm{p}+\mathrm{p}$ and $\mathrm{p}+\mathrm{Pb}$ collisions.

2.3.3. Glasma Graph. In the context of two-particle correlations, there are a total of eight topologies of the Glasma graph (full expression can be found in [31]); the contribution for one such diagram to the two-particle correlations can be written as

$$
\begin{gathered}
\frac{d^{2} N_{\text {Glasma- }-1}^{\text {corr. }}}{d^{2} \mathbf{p}_{T} d^{2} \mathbf{q}_{T} d y_{p} d y_{q}}=\frac{32 \alpha_{s}^{2}}{(2 \pi)^{10} \zeta N_{c} C_{F}^{3}} \frac{S_{\perp}}{\mathbf{p}_{T}^{2} \mathbf{q}_{T}^{2}} \\
\cdot \int_{\mathbf{k}_{T}} \Phi_{A}^{2}\left(\mathbf{k}_{T}\right) \Phi_{B}\left(\mathbf{p}_{T}-\mathbf{k}_{T}\right) \Phi_{B}\left(\mathbf{q}_{T}-\mathbf{k}_{T}\right) .
\end{gathered}
$$

Beyond the straightforward, perturbative result, the nonperturbative factor $\zeta$ has been introduced to account for the contributions to multiparticle production below the scale $Q_{s}^{2}$. Constraints from the independent analysis of $n$-particle multiplicity distributions [73,107-109] suggest that $\zeta$ be in the range $0.1-1$ and phenomenological studies typically employ the value of $\zeta=1 / 6$ obtained from $[108,109]$.

The collimation in the Glasma graph framework comes from the fact that each of the four UGDs in (14) has a bellshaped structure with a maximum corresponding to the saturation momentum. Such nature of the UGDs kinematically constrains two gluons to be produced in similar (or back-toback) directions giving rise to the double ridge structure in two-particle correlations $[27,110]$.

2.4. Dilute-Dense Models. Besides the perturbative diagrammatic approach taken in the Glasma graph calculation, several studies have been launched to investigate the origin of structure of the long range azimuthal correlations in the limit where a dilute projectile of individual partons scatter off the color fields of a dense projectile. Neglecting correlations of the incoming partons in the projectile, the double-inclusive distribution of the scattered partons takes the form $[37,39]$

$$
\begin{aligned}
& \frac{d^{2} N}{d y_{1} d^{2} \mathbf{p}_{1} d y_{2} d^{2} \mathbf{p}_{2}}=\int d^{2} \mathbf{b}_{1} d^{2} \mathbf{b}_{2} \int \frac{d^{2} \mathbf{k}_{1}}{(2 \pi)^{2}} \int \frac{d^{2} \mathbf{k}_{2}}{(2 \pi)^{2}} \\
& \cdot \int d^{2} \mathbf{r}_{1} d^{2} \mathbf{r}_{2} e^{i\left(\mathbf{p}_{1}-\mathbf{k}_{1}\right) \cdot \mathbf{r}_{1}} e^{i\left(\mathbf{p}_{2}-\mathbf{k}_{2}\right) \cdot \mathbf{r}_{2}} W_{q / g, y_{1}}\left(\mathbf{b}_{1}, \mathbf{k}_{1}\right) \\
& \cdot W_{q / g, y_{2}}\left(\mathbf{b}_{2}, \mathbf{k}_{2}\right)\left\langle\mathscr{D}\left(\mathbf{b}_{1}+\frac{\mathbf{r}_{1}}{2}, \mathbf{b}_{1}-\frac{\mathbf{r}_{1}}{2}\right)\right. \\
& \left.\cdot \mathscr{D}\left(\mathbf{b}_{2}+\frac{\mathbf{r}_{2}}{2}, \mathbf{b}_{2}-\frac{\mathbf{r}_{2}}{2}\right)\right\rangle,
\end{aligned}
$$




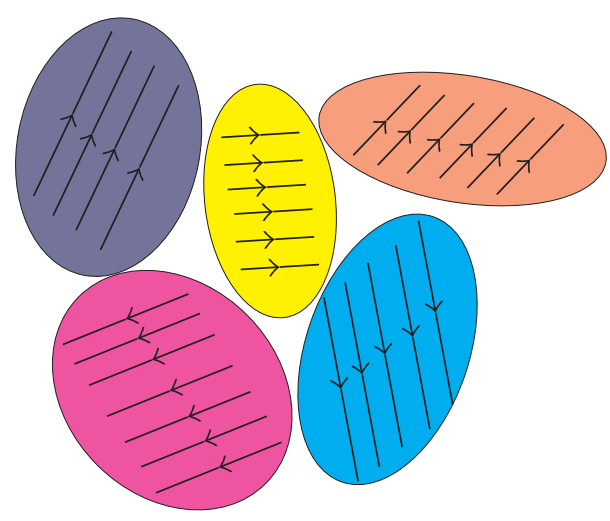

FIGURE 6: Color fields of the target are locally organized in domains of characteristic size of the inverse saturation scale $1 / Q_{s}$, taken from [39].

where $W_{q / g, y_{1}}\left(\mathbf{b}_{1}, \mathbf{k}_{1}\right)$ denotes the Wigner function of quarks/gluons inside the projectile and $\mathscr{D}(\mathbf{x}, \mathbf{y})=(1 /$ $\left.N_{c}\right) \operatorname{Tr}\left[V(\mathbf{x}) V^{\dagger}(\mathbf{y})\right]$ is the usual dipole-correlator of light-like Wilson lines in the fundamental/adjoint representation. While the transverse momenta $\mathbf{k}_{1}$ and $\mathbf{k}_{2}$ of the two incoming quarks are uncorrelated, it is evident from (15) that the correlations in the momentum transfers $\mathbf{p}_{1}-\mathbf{k}_{1}$ and $\mathbf{p}_{2}-\mathbf{k}_{2}$ give rise to azimuthal correlations of the scattered partons $\mathbf{p}_{1}$ and $\mathbf{p}_{2}$. We note that, in contrast to the perturbative calculation, present calculations in the dilutedense description usually do not take into account rapidity evolution between the produced particles and are therefore limited to the kinematic range where $y_{1}-y_{2} \ll 1 / \alpha_{s}$ where evolution effects can be neglected. While recent progress has been made in formulating evolution equations for multiparticle production in dilute-dense systems [111], these have not been employed for phenomenological studies so far.

By evaluating the dipole operator in short-distance expansion $\left(Q_{s}|\mathbf{x}-\mathbf{y}| \ll 1\right)$

$$
\begin{aligned}
& \mathscr{D}(\mathbf{x}, \mathbf{y}) \\
& \quad \simeq 1-\frac{(x-y)^{i}(x-y)^{j}}{4 N_{c}} E_{i}^{a}\left(\frac{\mathbf{x}+\mathbf{y}}{2}\right) E_{j}^{a}\left(\frac{\mathbf{x}+\mathbf{y}}{2}\right),
\end{aligned}
$$

where $E_{i}(\mathbf{x})=i V(\mathbf{x}) \partial_{i} V^{\dagger}(\mathbf{x})$ denotes the light-cone electric field, one can establish an intuitive picture of the origin of the long range near-side correlations [24]. When a projectile parton scatters off the color field of the nucleus, it receives a transverse momentum kick in the direction of the colorelectric field of the target. Color fields fluctuate from event to event and are locally organized in domains of size $\sim 1 / Q_{s}$ as illustrated in Figure 6. When two (or more) quarks scatter off the same domain, they will receive a similar kick whenever they are in the same color state. Naturally, this leads to a correlation which is suppressed by $1 / N_{c}^{2}$ (in the limit of large $N_{c}$ ) and by the number of domains $Q_{s}^{2} S_{\perp}$, where $S_{\perp}$ denotes the transverse area probed by the projectile.

Several studies have computed the azimuthal harmonics of the two-particle correlation function in (15), either based directly on numerical evaluations including JIMWLK evolution $[37,39]$ or within (semi)analytic models [33, 112, 113]. Generally, the double-inclusive spectrum in (15) features sizable azimuthal correlations $v_{n}$ which are on the order of $1 / \sqrt{N_{c}^{2}-1}$ and most pronounced when both parton momenta $p_{1}$ and $p_{2}$ are on the order of the saturation scale. While the double-inclusive spectrum for the two incoming quarks features both even $(n=2,4, \ldots)$ and odd $(n=$ $1,3,5, \ldots)$ harmonics, it turns out that the odd harmonics for gluons vanish identically due to an exact symmetry of the the correlation function under $\mathbf{p}_{1} \rightarrow-\mathbf{p}_{1}$. Even though the dilute-dense framework described above certainly does not provide the most realistic initial-state model for midrapidity particle production in high-multiplicity events, it turns out that the absence of odd harmonics for gluons poses a more general problem within the CGC framework. However, as discussed in Section 3, recent simulations including the earlytime classical Yang-Mills dynamics have been able to resolve this puzzle to some extent.

2.5. Classical Yang-Mills. Beyond the approaches outlined above, there have also been new theoretical developments in the study of initial-state correlations in event-by-event simulations in classical Yang-Mills theory [38, 72, 73]. In this approach, (5) is solved numerically for the individual colliding hadrons or nuclei in Lorentz gauge $\partial_{\mu} A^{\mu}=0$, where

$$
A_{A(B)}^{ \pm}=-\frac{\rho_{A(B)}\left(x^{\mp}, \mathbf{x}_{\perp}\right)}{\nabla_{\perp}^{2}+m^{2}},
$$

and then transformed into the light-cone gauge $A^{+}\left(A^{-}\right)=0$, where one finds [114-119]

$$
\begin{aligned}
A_{A(B)}^{i}\left(\mathbf{x}_{\perp}\right) & =\theta\left(x^{-}\left(x^{+}\right)\right) \frac{i}{g} V_{A(B)}\left(\mathbf{x}_{\perp}\right) \partial_{i} V_{A(B)}^{\dagger}\left(\mathbf{x}_{\perp}\right), \\
A^{-}\left(A^{+}\right) & =0 .
\end{aligned}
$$

The infrared regulator $m$ in (17) is of order $\Lambda_{\mathrm{QCD}}$ and crudely incorporates color confinement at the nucleon level by damping the Coulomb tail. The gauge field in the forward light-cone after the collision at time $\tau=0$ is given by the solution of the CYM equations in Fock-Schwinger gauge $A^{\tau}=\left(x^{+} A^{-}+x^{-} A^{+}\right) / \tau=0$ in terms of the gauge fields of the colliding nuclei $[120,121]$ :

$$
\begin{aligned}
A^{i} & =A_{(A)}^{i}+A_{(B)}^{i}, \\
A^{\eta} & =\frac{i g}{2}\left[A_{(A)}^{i}, A_{(B)}^{i}\right], \\
\partial_{\tau} A^{i} & =0, \\
\partial_{\tau} A^{\eta} & =0 .
\end{aligned}
$$

Such gluon fields produced after the collision are evolved in time according to Classical Yang-Mills equations up to time $\tau \sim 1 / Q_{s}$ to estimate the gluon spectrum $d N_{g} / d y d^{2} k_{t}$ by 
imposing Coulomb gauge $\left.\partial_{i} A^{i}\right|_{\tau}=0$ and extracting the equal time correlation function $[122,123]$ :

$$
\begin{aligned}
& \left.\frac{d N}{d^{2} k_{t} d y}\right|_{\tau} \\
& =\frac{1}{(2 \pi)^{2}} \sum_{\lambda, a}\left|\tau g^{\mu \nu}\left(\xi_{\mu}^{\lambda, k_{t} *}(\tau) \overleftrightarrow{\partial_{\tau}} A_{\nu}^{a}\left(\tau, k_{t}\right)\right)\right|^{2},
\end{aligned}
$$

where $g^{\mu \nu}=\left(1,-1,-1,-\tau^{-2}\right)$ denotes the Bjorken metric and $\lambda=1,2$ labels the two transverse polarizations. In Coulomb gauge, the mode functions take the form

$$
\begin{aligned}
& \xi_{\mu}^{(1), k_{t}}(\tau)=\frac{\sqrt{\pi}}{2\left|k_{t}\right|}\left(\begin{array}{c}
-k_{y} \\
k_{x} \\
0
\end{array}\right) H_{0}^{(2)}\left(\left|k_{t}\right| \tau\right), \\
& \xi_{\mu}^{(2), k_{t}}(\tau)=\frac{\sqrt{\pi}}{2\left|k_{t}\right|}\left(\begin{array}{c}
0 \\
0 \\
k_{T} \tau
\end{array}\right) H_{1}^{(2)}\left(\left|k_{t}\right| \tau\right),
\end{aligned}
$$

where $H_{\alpha}^{(2)}$ denote the Hankel functions of the second-type and order $\alpha$ (see [120] for details).

The calculations in the framework of classical YangMills naturally include the Glasma graphs and extend the reach of perturbative calculations towards lower $p_{T}$ by consistently including multiple-scattering effects as well as coherent rescattering in the final-state. Since event-by-event simulations in the classical Yang-Mills theory also allow for an improved treatment of the impact parameter dependence, they can be used in the future to systematically study initialstate effects across different collision geometries, for example, in $\mathrm{p}+\mathrm{A}, \mathrm{d}+\mathrm{A}$, and ${ }^{3} \mathrm{He}+\mathrm{A}$ collisions at RHIC $[10,11,122]$.

2.6. Hadronization. So far, we have outlined the computation of initial-state correlations at the parton level. The mechanism of hadronization converts the partonic correlations driven by initial-state dynamics into correlated production of final-state particles. Implementation of a realistic scheme of hadronization is therefore essential for the phenomenology of small systems collisions. A first-principle QCD based approach to such a problem is very challenging. One therefore resorts to several available approximation schemes for fragmentations of partons. The most commonly used approach is the standard parton-hadron independent hadronization scheme [123] in which, for example, the single-inclusive hadron distributions are obtained by convoluting the gluon distributions with fragmentation functions as

$$
\frac{d N_{h}}{d^{2} \mathbf{p}_{T} d y}=\int_{z_{\min }}^{1} \frac{d z}{z^{2}} \frac{d N_{g}}{d^{2} \mathbf{q}_{T} d y} D_{g \rightarrow h}\left(z=\frac{\mathbf{p}_{T}}{\mathbf{q}_{T}}, \mu^{2}\right),
$$

where $D_{g \rightarrow h}\left(z, \mu^{2}\right)$ denotes the probability that a gluon fragments into a hadron carrying $z$ fraction of its momentum at a scale $\mu^{2}$. The lower limit of the integral is determined from the kinematic requirement that the momentum fraction of the gluons $x \leq 1$. Commonly used forms for $D_{g \rightarrow h}\left(z, \mu^{2}\right)$ such as KKP or DSS fragmentation functions are obtained from fits to the inclusive hadron production data in $\mathrm{e}^{+}+\mathrm{e}^{-}$ and $p+p$ collisions $[124,125]$. In case of double-inclusive production relevant for the study of two-particle correlations, one assumes an ansatz of the form [27]

$$
\begin{aligned}
& \frac{d^{2} N_{h}^{\text {corr. }}}{d^{2} p_{T} d^{2} q_{T} d \eta_{p} d \eta_{q}}=\int_{z_{\min }}^{1} d z_{1} d z_{2} \\
& \cdot \frac{D\left(z_{1}\right)}{z_{1}^{2}} \frac{D\left(z_{2}\right)}{z_{2}^{2}} \frac{d^{2} N_{g}^{\text {corr. }}}{d^{2} \mathbf{p}_{T} d^{2} \mathbf{q}_{T} d \eta_{p} d \eta_{q}}\left(\frac{p_{T}}{z_{1}}, \frac{q_{T}}{z_{2}}\right) .
\end{aligned}
$$

A limitation of this standard approach of hadronization is that the applicability of the fragmentations functions is questionable at low virtuality $\mu^{2}$. Therefore, such scheme of hadronization can not be used for bulk particle production which is dominated by soft processes with typical virtuality scale $\mu^{2} \lesssim 1 \mathrm{GeV}$. In this regime, an alternative approach such as the Local Parton-Hadron Duality (LHPD) [126] can be used to describe bulk particle production driven by initial sate dynamics [127].

The state-of-the-art scheme of hadronization to describe bulk particle production used in several event generators like PYTHIA $[128,129]$ is based on the Monte-Carlo implementation of the Lund string fragmentation function:

$$
f\left(z, m_{T}\right)=\frac{1}{z}(1-z)^{a} \exp \left(-\frac{b m_{T}^{2}}{z}\right),
$$

where $m_{T}$ and $z$ denote the transverse mass and the lightcone momentum fraction of the fragmenting hadron. The default parameters $a$ and $b$ are constrained by global analysis of $\mathrm{p}+\mathrm{p}$ data. The hadronization scheme in PYTHIA also includes decays of hadron resonances and final-state hadronic interactions. A quantitative study of the effect of hadronization on initial-state parton level correlations using different schemes of fragmentation has been done in [130]. The study indicates that the structure of the final azimuthal correlations is very much sensitive to the choice of fragmentation. For example, the resonance decay and hadronic interactions can distort the initial-state correlations and introduce artificial final-state correlations in the produced hadrons. Such mechanism will complicate the interpretation of experimental data. In such a context, it has been demonstrated that hadronization effects in PYTHIA combined with the scheme of color reconnection produce effects that can mimic collective flow like pattern leading to a strong mass ordering of $\left\langle p_{T}\right\rangle$ [131] in $\mathrm{p}+\mathrm{p}$ collisions. In addition, the hadronic transport models that implement the Lund string fragmentation of PYTHIA have also been shown to qualitatively reproduce the systematics of $v_{2}$ measured in $\mathrm{p}+\mathrm{Pb}$ collisions [132]. Clearly, more phenomenology in this direction will further improve our understanding. Meanwhile, it is essential to implement a state-of-the-art framework of hadronization to test different features of multiparticle correlations in the CGC at the level of hadrons; work in this direction is in progress [133]. 


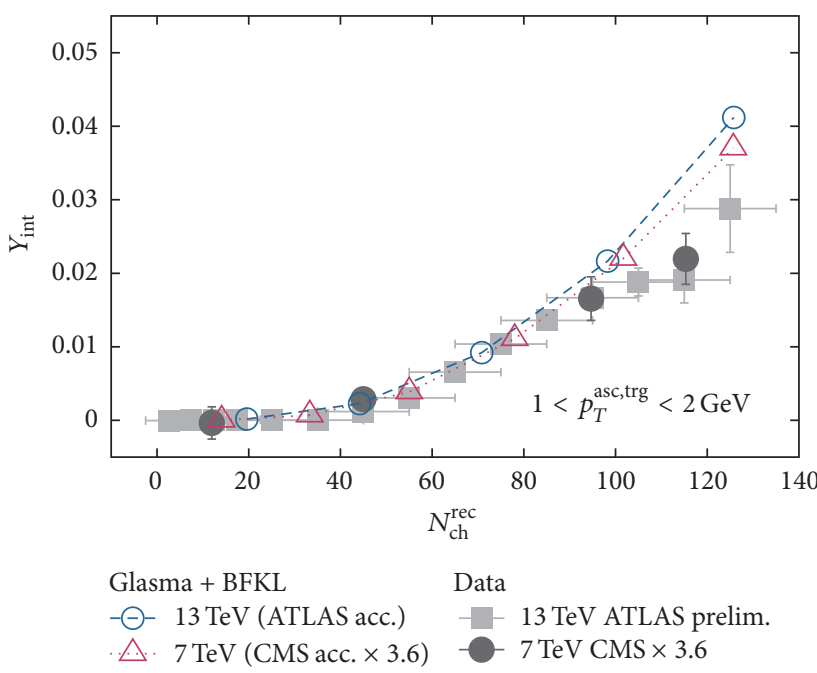

(a)

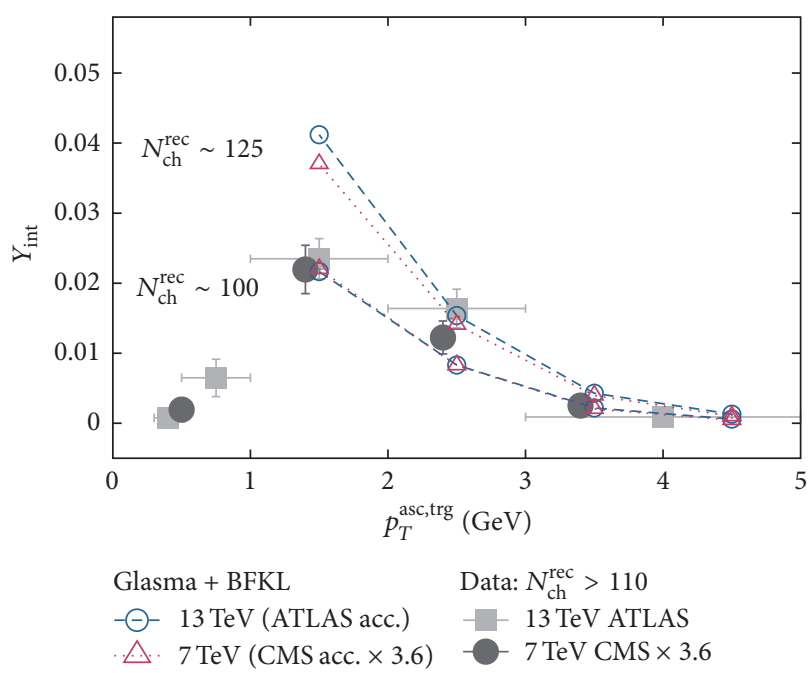

(b)

FIGURE 7: (a) The energy independence of the near-side ridge yield in $p+p$ collisions obtained from [40]; (b) transverse momentum dependence of the same quantity shown for two energies. The model calculations are compared to data from the CMS and the ATLAS collaborations.

\section{Comparison with the Latest Experimental Observations}

3.1. $p+p$ Collisions. We begin with a comparison of model calculations with the latest data in $\mathrm{p}+\mathrm{p}$ collisions at LHC energies [12], where the quantity of experimental interest is the rapidity and momentum integrated correlation function defined as

$$
\begin{aligned}
\frac{d N}{d \Delta \phi} & =\int_{p_{T}^{\min }}^{p_{T}^{\max }} \frac{d p_{T}^{2}}{2} \int_{q_{T}^{\min }}^{q_{T}^{\max }} \frac{d q_{T}^{2}}{2} \int d \phi_{p} \int d \phi_{q} \delta\left(\phi_{p}-\phi_{q}\right. \\
-\Delta \phi) & \frac{d^{2} N^{\mathrm{corr} .}}{d^{2} \mathbf{p}_{T} d^{2} \mathbf{q}_{T} d \eta_{p} d \eta_{q}}\left(\frac{p_{T}}{z_{1}}, \frac{q_{T}}{z_{2}}, \Delta \phi\right)
\end{aligned}
$$

which so far has only been computed in the perturbative CGC approach. The quantity of experimental interest is the nearside yield $Y_{\text {int }}$ defined as the ZYAM subtracted integrated associated yield per trigger given by

$$
Y_{\mathrm{int}}=\frac{1}{N_{\text {trig }}} \int_{0}^{\Delta \phi_{\min }} d \Delta \phi\left(\frac{d N}{d \Delta \phi}-\left.\frac{d N}{d \Delta \phi}\right|_{\Delta \phi_{\min }}\right) .
$$

Here, $\Delta \phi=\Delta \phi_{\min }$ corresponds to the minimum of the dihadron correlation function (ZYAM) and $N_{\text {trig }}$ is the number of trigger particles. A quantitative comparison of the near-side yield in $\mathrm{p}+\mathrm{p}$ collisions shown in Figure 7 yields a good agreement between initial-state calculations [40] and experimental data at both 7 and $13 \mathrm{TeV}$. The striking feature of the data model comparison shown in Figure 7 is that the near-side yield is approximately energy independent. Such energy independent scaling is naturally explained within the framework of CGC [40]. Origin of such scaling can be understood as follows. In the CGC framework, a single scale $Q_{s}^{2}$ determines both the single and double-inclusive production in $\mathrm{p}+\mathrm{p}$ collisions. The dependence on the center of mass energy in both multiplicity $N_{\mathrm{ch}}^{\mathrm{rec}}$ and the near-side yield $Y_{\text {int }}$ enters only through $Q_{s}^{2}$; that is,

$$
\begin{gathered}
N_{\mathrm{ch}}^{\mathrm{rec}}\left(Q_{0}^{2}, \sqrt{s}\right)=N_{\mathrm{ch}}^{\mathrm{rec}}\left(Q_{s}^{2}\right), \\
Y_{\text {int }}\left(Q_{0}^{2}, \sqrt{s}\right)=Y_{\text {int }}\left(Q_{s}^{2}\right),
\end{gathered}
$$

where $Q_{s}^{2} \equiv Q_{s}^{2}\left(Q_{0}^{2}, \sqrt{s}\right)$. Fixing the multiplicity $N_{\text {ch }}^{\text {rec }}\left(Q_{s}^{2}\right)$ therefore naturally fixes the value of $Y_{\text {int }}\left(Q_{s}^{2}\right)$ giving energy independent scaling of the ridge-like correlations. Such scaling provides strong indication of multiparticle production driven by a single semihard scale as captured in the CGC.

3.2. $p+A$ Collisions. A detailed quantitative estimation of the dihadron correlations in $\mathrm{p}+\mathrm{Pb}$ collisions at the LHC using the perturbative dijet and Glasma graph framework has been performed in [31]. One of the interesting results from [31] shown in Figure 8(a) indicates that the CGC framework naturally explains the increase in the near-side yield in $\mathrm{p}+\mathrm{Pb}$ collisions as compared to $\mathrm{p}+\mathrm{p}$ collisions for events with the same multiplicity measured by the CMS collaboration. Results shown in Figure 8(b) indicate that such framework also very well reproduces the peripheral subtracted correlation function $\left(1 / N_{\text {trig }}\right) d N / d \Delta \phi$ measured by the ALICE collaboration in $\mathrm{p}+\mathrm{Pb}$ collisions. Aforementioned due to the limitations of the perturbative framework, such estimations can be performed only for trigger and associated momentum $\mathbf{p}_{T}, \mathbf{q}_{T} \geq 1 \mathrm{GeV}$.

Recent simulations in the framework of classical YangMills that go beyond such limitations of the perturbative regime have already opened the path for improved phenomenology. Even though at present these calculations do not yet include dijet graphs (note that also the interference contribution between Glasma graphs and jet graphs no 


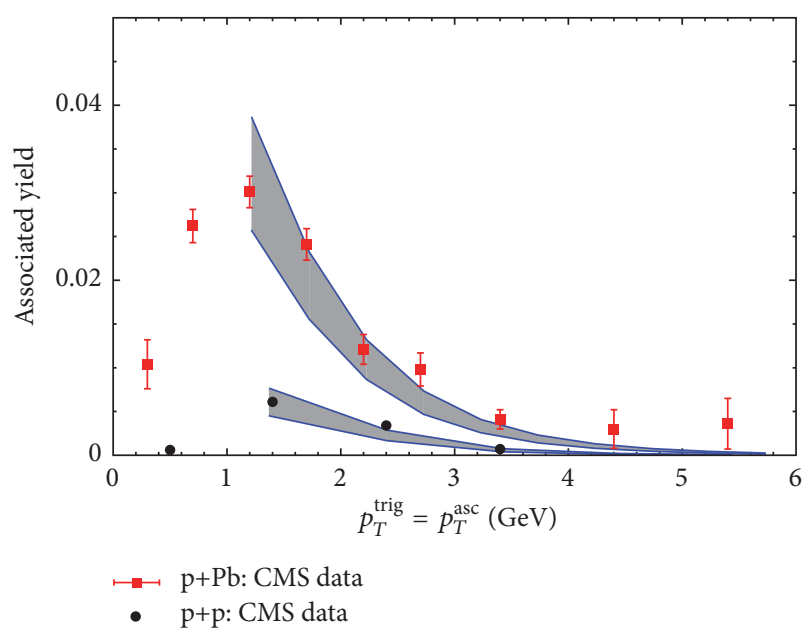

(a)

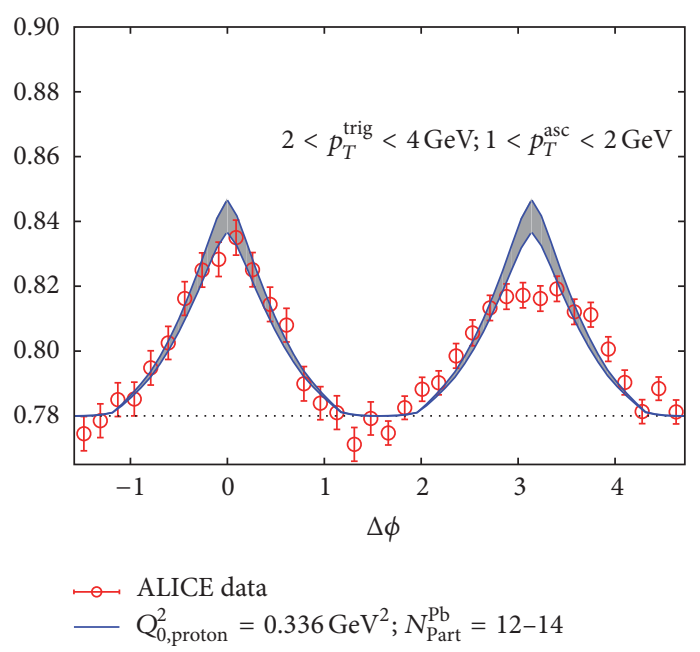

(b)

Figure 8: Figure showing the systematics of ridge-like correlations in $\mathrm{p}+\mathrm{p}$ and $\mathrm{p}+\mathrm{Pb}$ collisions captured in the Glasma graph framework taken from [31]. The comparisons are made with the CMS data for $\mathrm{p}+\mathrm{p}$ and $\mathrm{p}+\mathrm{Pb}$ collisions for events having $N_{\text {trk }}^{\text {offline }} \geq 110$ from [5,6] and ALICE data for $\mathrm{p}+\mathrm{Pb}$ collisions [7].

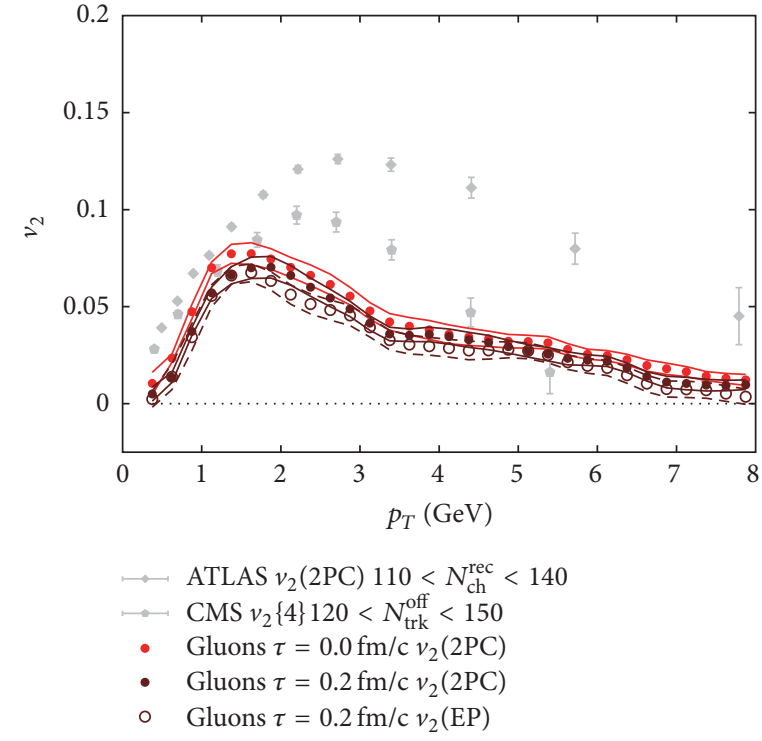

(a)

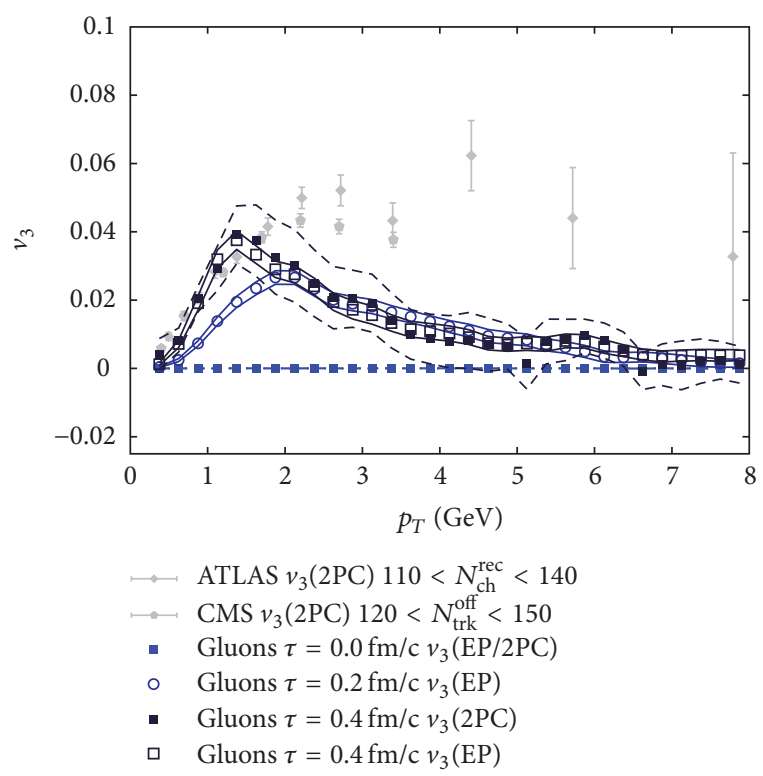

(b)

FIGURE 9: Gluon $v_{2}\left(p_{T}\right)$ (a) and $v_{3}\left(p_{T}\right)(\mathrm{b})$ in $\mathrm{p}+\mathrm{Pb}$ collisions from Yang-Mills simulations, figure obtained from [38] compared to data from ATLAS [63] and CMS collaboration [64] for inclusive hadrons.

longer vanishes beyond leading order) and hadronization effects and thus do not allow for a direct comparison with experimental data, simulations in this regard have led to new insight into the correlations at the parton level concerning in particular the dynamics of the correlations during the very early stages. The results from the recent classical Yang-Mills simulations performed in $\mathrm{p}+\mathrm{Pb}$ collisions [38] are shown in Figure 9. While gluons are produced with a significant momentum space anisotropy at $\tau=0^{+}$, initially the two-particle correlation function is symmetric around $\Delta \phi=$ $\pi / 2$ and features only even harmonics in accordance with the perturbative result [38]. However, including the effects of the classical Yang-Mills evolution up to $\tau=0.4 \mathrm{fm} / \mathrm{c}$ leads to the buildup of a sizable $v_{3}$ on the parton level, while the initial-state $v_{2}$ remains intact [38]. These results indicate that at least at the gluon level the nonperturbative dynamics of the Glasma fields gives rise to large values of both $v_{2}$ and $v_{3}$ at time scales $\tau \sim 1 / Q_{s}$ after the collision that are comparable 
to what is seen in the data. Interestingly, sizable $v_{2}$ and $v_{3}$ in this framework also extend to large transverse momentum probably beyond the applicability of hydrodynamics in $\mathrm{p}+\mathrm{Pb}$ collisions [134].

While a systematic comparison of the initial-state calculations to experimental data in $\mathrm{p}+\mathrm{Pb}$ collisions yields a similar level of quantitative agreement of two-particle correlations for momenta $p_{T}>1 \mathrm{GeV}[29,31]$, there has been enormous progress on the experimental side to identify additional signatures of collective motion which could be indicative of the onset of significant final-state effects. Even though some observables, such as mass ordering properties observed in correlations between identified hadrons [135], are not necessarily sensitive to the origin of the correlations, more promising directions including, for example, correlations between more than two particles have also been explored [136, 137]. One of the most striking observations in this regard is the sign change of the four-particle cumulant $c_{2}\{4\}$ observed around $N_{\mathrm{ch}}\left(\left|\eta_{\mathrm{lab}}\right|<1\right) \sim 60$ by ALICE [137]. However, most of the recent measurements have focused on low $p_{T}$ observables where the perturbative calculations [27-29, 31, 40] outlined in Section 2.3 do not necessarily apply, hence complicating the comparison between theory and experiment.

Nevertheless, there have been first attempts to extend the theoretical framework to understand whether certain features of the low $p_{T}$ data such as the sign change of $c_{2}\{4\}$ can also be explained from initial-state effects. Based on the dilute-dense approximation in (16) first attempts have been made to study initial-state correlations of more than two particles. Generalizing the dilute-dense formalism to $n$ particle scattering, it was pointed out in $[35,39,138]$ that the four-particle cumulant $c_{2}\{4\}$ is sensitive to non-Gaussian correlations of the color-electric fields inside the target. While the lowest order perturbative contribution to $c_{2}\{4\}$ was found to be positive, it was argued [35] that non-Gaussian correlations of the domains of color-electric fields may explain the experimentally observed sign change of $c_{2}\{4\}$ as a function of multiplicity. Even though a dynamical explanation for the existence of sizable non-Gaussian correlations is still lacking, this topic is an active subject of further investigations. However, one should also caution that in contrast to the twoparticle cumulant $c_{2}\{2, \mid \Delta \eta>2\}$, where short-range correlations are suppressed by introducing a large rapidity gap, the four-particle cumulant $c_{2}\{4\}$ also receives contributions from short-range correlations and interference diagrams. While a complete theoretical calculation of higher-order $n$-particle correlations is desirable, further progress is needed to tackle this problem.

Similarly, preliminary results from event-by-event simulations in classical Yang-Mills theory a la [38] also suggest a negative sign of $c_{2}\{4\}$ when all particles have low momenta, while at high $p_{T}$ the four-particle cumulant is always positive [139]. While the first results are interesting, further theoretical progress is needed to decide unambiguously whether the sign change in $c_{2}\{4\}$ can be explained in terms of initialstate and early-time effects. It would also be interesting to extend the experimental measurements of higher cumulants towards higher momenta to achieve convergence on this issue.

\section{Summary}

Experimental observations of collective correlations in small systems challenge our current understanding of the spacetime evolution of high-energy collisions. While at low multiplicities one expects dominance of initial-state effects, it is also expected that at sufficiently high multiplicities initialstate correlations are destroyed due to final-state interactions and the hydrodynamic response to the initial-state geometry provides the dominant source of correlations. However, it is theoretically challenging to predict the transition from initial-state to final-state dominated dynamics pointing to the importance of developing a unified theoretical framework where both effects are consistently taken into account.

In this brief review, we specifically outlined recent developments in the approach based on initial-state dynamics. While calculations based purely on initial-state correlations are able to quantitatively describe various features of the experimental data in $\mathrm{p}+\mathrm{p}$ and $\mathrm{p}+\mathrm{Pb}$ collisions up to the highest multiplicity windows, a simultaneous description of the low and high $p_{T}$ observables over a wide range of multiplicity remains challenging within any single theoretical framework. Of course, several outstanding issues remain on the theory side and the development of a unified framework that combines (1) dynamics of multiple-soft interactions with (2) a first-principle calculation of jet production and (3) a state-of-the-art fragmentation scheme is essential for a wide range of phenomenological applications. So far, experimental efforts have focused on soft observables such as $v_{n}$ s; however, complimentary information on the dynamics can be obtained by considering higher-momentum probes. While in heavy-ion collisions the observation of strong jetquenching phenomena provides an important indication for the formation of a strongly interacting Quark-Gluon plasma, no such features have been reported so far in small systems. Even though such an analysis is experimentally challenging, it would be important to establish to what extent minijets and actual jets are modified in high-multiplicity events to further constrain the relative importance of initial-state and finalstate effects in small systems.

\section{Competing Interests}

The authors declare that there is no conflict of interests regarding the publication of this paper.

\section{Acknowledgments}

The authors thank Kevin Dusling, Ulrich Heinz, Tuomas Lappi, Wei Li, Derek Teaney, and Raju Venugopalan for important discussions. The authors thank Bjoern Schenke for careful reading of the manuscript and helpful comments and suggestions. The authors are supported under Department of Energy Contract no. DE-SC0012704. Sören Schlichting acknowledges support under DOE Grant no. DE-FG0297ER41014. 


\section{References}

[1] I. Arsene, I. G. Bearden, D. Beavis et al., "Quark-gluon plasma and color glass condensate at RHIC? The perspective from the BRAHMS experiment," Nuclear Physics A, vol. 757, no. 1-2, pp. 1-27, 2005.

[2] B. B. Back, M. D. Baker, M. Ballintijn et al., "The PHOBOS perspective on discoveries at RHIC," Nuclear Physics A, vol. 757, no. 1-2, pp. 28-101, 2005.

[3] J. Adams, M. M. Aggarwal, Z. Ahammed et al., "Experimental and theoretical challenges in the search for the quark-gluon plasma: the STAR Collaboration's critical assessment of the evidence from RHIC collisions," Nuclear Physics A, vol. 757, no. 1-2, pp. 102-183, 2005.

[4] K. Adcox, S. S. Adler, S. Afanasiev et al., "Formation of dense partonic matter in relativistic nucleus-nucleus collisions at RHIC: experimental evaluation by the PHENIX Collaboration," Nuclear Physics A, vol. 757, no. 1-2, pp. 184-283, 2005.

[5] V. Khachatryan, A. M. Sirunyan, A. Tumasyan et al., "Observation of long-range, near-side angular correlations in protonproton collisions at the LHC," Journal of High Energy Physics, vol. 2010, article 91, 2010.

[6] S. Chatrchyan, V. Khachatryan, A. M. Sirunyan et al., "Observation of long-range, near-side angular correlations in $\mathrm{pPb}$ collisions at the LHC," Physics Letters B, vol. 718, no. 3, pp. 795814, 2013.

[7] B. Abelev and ALICE Collaboration, "Long-range angular correlations on the near and away side in $\mathrm{p}-\mathrm{Pb}$ collisions at $\sqrt{s_{N N}}=5.02$ TeV," Physics Letters B, vol. 719, no. 1-3, pp. 2941, 2013.

[8] G. Aad and ATLAS Collaboration, "Observation of associated near-side and away-side long-range correlations in $\sqrt{s_{N N}}=$ $5.02 \mathrm{TeV}$ proton-lead collisions with the ATLAS detector," Physical Review Letters, vol. 110, no. 18, Article ID 182302, 2013.

[9] A. Adare, C. Aidala, N. N. Ajitanand et al., "Measurement of long-range angular correlation and quadrupole anisotropy of pions and (anti)protons in central $d+A u$ collisions at $\sqrt{s_{\mathrm{NN}}}=$ 200 GeV," Physical Review Letters, vol. 114, no. 19, Article ID 192301, 2015.

[10] L. Adamczyk, J. K. Adkinsu, G. Agakishiev et al., "Long-range pseudorapidity dihadron correlations in $d+$ Au collisions at $\sqrt{s_{N N}}=200 \mathrm{GeV}$," Physics Letters B, vol. 747, pp. 265-271, 2015.

[11] A. Adare, S. Afanasiev, C. Aidala et al., "Measurements of elliptic and triangular flow in high-multiplicity ${ }^{3} \mathrm{He}+\mathrm{Au}$ collisions at $\sqrt{s_{N N}}=200 \mathrm{GeV}$," Physical Review Letters, vol. 115, no. 14, Article ID 142301, 2015.

[12] G. Aad, B. Abbott, J. Abdallah et al., "Observation of long-range elliptic azimuthal anisotropies in $\sqrt{s}=13$ and $2.76 \mathrm{TeV} p p$ collisions with the ATLAS detector," Physical Review Letters, vol. 116, no. 17, Article ID 172301, 20 pages, 2016.

[13] V. Khachatryan, A. M. Sirunyan, A. Tumasyan et al., "Measurement of long-range near-side two-particle angular correlations in pp collisions at $\sqrt{s}=13$ TeV," Physical Review Letters, vol. 116, Article ID 172302, 2016.

[14] P. Bozek and W. Broniowski, "Collective dynamics in highenergy proton-nucleus collisions," Physical Review C, vol. 88, no. 1, Article ID 014903, 2013.

[15] P. Bozek, W. Broniowski, and G. Torrieri, "Mass hierarchy in identified particle distributions in proton-lead collisions," Physical Review Letters, vol. 111, no. 17, Article ID 172303, 2013.
[16] A. Bzdak, B. Schenke, P. Tribedy, and R. Venugopalan, "Initialstate geometry and the role of hydrodynamics in protonproton, proton-nucleus, and deuteron-nucleus collisions," Physical Review C, vol. 87, no. 6, Article ID 064906, 2013.

[17] G.-Y. Qin and B. Muller, "Elliptic and triangular flow anisotropy in deuteron-gold collisions at $\sqrt{s_{N N}}=200 \mathrm{GeV}$ at RHIC and in proton-lead collisions at $\sqrt{s_{N N}}=5.2 \mathrm{TeV}$ at the LHC," Physical Review C, vol. 89, no. 4, Article ID 044902, 2014.

[18] K. Werner, M. Bleicher, B. Guiot, I. Karpenko, and T. Pierog, "Evidence for flow from hydrodynamic simulations of $p-\mathrm{Pb}$ collisions at $5.02 \mathrm{TeV}$ from $v_{2}$ mass splitting," Physical Review Letters, vol. 112, Article ID 232301, 2014.

[19] B. Schenke and R. Venugopalan, "Eccentric protons? Sensitivity of flow to system size and shape in $p+p, p+\mathrm{Pb}$ and $\mathrm{Pb}+\mathrm{Pb}$ collisions," Physical Review Letters, vol. 113, Article ID 102301, 2014.

[20] A. Bzdak and G.-L. Ma, "Elliptic and triangular flow in $p$-Pb and peripheral $\mathrm{Pb}-\mathrm{Pb}$ collisions from parton scatterings," Physical Review Letters, vol. 113, no. 25, Article ID 252301, 2014.

[21] T. Kalaydzhyan and E. Shuryak, "Collective flow in highmultiplicity proton-proton collisions," Physical Review C, vol. 91, no. 5, Article ID 054913, 5 pages, 2015.

[22] E. Shuryak and I. Zahed, "High-multiplicity $p p$ and $p A$ collisions: hydrodynamics at its edge," Physical Review C, vol. 88, no. 4, Article ID 044915, 2013.

[23] P. Ghosh, S. Muhuri, J. K. Nayak, and R. Varma, "Indication of transverse radial flow in high-multiplicity proton-proton collisions at the Large Hadron Collider," Journal of Physics G: Nuclear and Particle Physics, vol. 41, no. 3, Article ID 035106, 2014.

[24] A. Kovner and M. Lublinsky, "Angular correlations in gluon production at high energy," Physical Review D, vol. 83, no. 3, Article ID 034017, 12 pages, 2011.

[25] A. Kovner and M. Lublinsky, "Angular correlations and high energy evolution," Physical Review D, vol. 84, Article ID 094011, 2011.

[26] E. Levin and A. H. Rezaeian, "Ridge from the BFKL evolution and beyond," Physical Review D, vol. 84, Article ID 034031, 2011.

[27] K. Dusling and R. Venugopalan, "Azimuthal collimation of long range rapidity correlations by strong color fields in high multiplicity hadron-hadron collisions," Physical Review Letters, vol. 108, no. 26, Article ID 262001, 5 pages, 2012.

[28] K. Dusling and R. Venugopalan, "Evidence for BFKL and saturation dynamics from dihadron spectra at the LHC," Physical Review D, vol. 87, no. 5, Article ID 051502, 2013.

[29] K. Dusling and R. Venugopalan, "Explanation of systematics of CMS p + Pb high multiplicity dihadron data at $\sqrt{s_{N N}}=5.2 \mathrm{TeV}$," Physical Review D, vol. 87, no. 5, Article ID 054014, 2013.

[30] Y. V. Kovchegov and D. E. Wertepny, "Long-range rapidity correlations in heavy-light ion collisions," Nuclear Physics A, vol. 906, pp. 50-83, 2013.

[31] K. Dusling and R. Venugopalan, "Comparison of the color glass condensate to dihadron correlations in proton-proton and proton-nucleus collisions," Physical Review D, vol. 87, no. 9, Article ID 094034, 2013.

[32] Y. V. Kovchegov and D. E. Wertepny, "Two-gluon correlations in heavy-light ion collisions: energy and geometry dependence, IR divergences, and $\mathrm{k}_{T}$-factorization," Nuclear Physics A, vol. 925, pp. 254-295, 2014.

[33] A. Dumitru and A. V. Giannini, "Initial state angular asymmetries in high energy $\mathrm{p}+\mathrm{A}$ collisions: spontaneous breaking 
of rotational symmetry by a color electric field and C-odd fluctuations," Nuclear Physics A, vol. 933, pp. 212-228, 2015.

[34] A. Dumitru and V. Skokov, "Anisotropy of the semiclassical gluon field of a large nucleus at high energy," Physical Review D, vol. 91, no. 7, Article ID 074006, 7 pages, 2015.

[35] A. Dumitru, L. McLerran, and V. Skokov, "Azimuthal asymmetries and the emergence of 'collectivity' from multi-particle correlations in high-energy pA collisions," Physics Letters B, vol. 743, pp. 134-137, 2015.

[36] M. Gyulassy, P. Levai, I. Vitev, and T. S. Biró, "Non-Abelian bremsstrahlung and azimuthal asymmetries in high energy $p+$ A reactions," Physical Review D, vol. 90, no. 5, Article ID 054025 , 16 pages, 2014.

[37] T. Lappi, "Azimuthal harmonics of color fields in a high energy nucleus," Physics Letters B, vol. 744, pp. 315-319, 2015.

[38] B. Schenke, S. Schlichting, and R. Venugopalan, "Azimuthal anisotropies in $\mathrm{p}+\mathrm{Pb}$ collisions from classical Yang-Mills dynamics," Physics Letters B, vol. 747, pp. 76-82, 2015.

[39] T. Lappi, B. Schenke, S. Schlichting, and R. Venugopalan, "Tracing the origin of azimuthal gluon correlations in the color glass condensate," Journal of High Energy Physics, vol. 2016, article 61, 2016.

[40] K. Dusling, P. Tribedy, and R. Venugopalan, "Energy dependence of the ridge in high multiplicity proton-proton collisions," Physical Review D, vol. 93, no. 1, Article ID 014034, 2016.

[41] X.-N. Wang and M. Gyulassy, "Transverse flow due to minijets in pp collisions at $\sqrt{ } s=1.8 \mathrm{TeV}$," Physics Letters $B$, vol. 282, no. 3-4, pp. 466-470, 1992.

[42] P. Lévai and B. Müller, "Transverse baryon flow as possible evidence for a quark-gluon-plasma phase," Physical Review Letters, vol. 67, no. 12, pp. 1519-1522, 1991.

[43] J. Adams, C. Adler, M. M. Aggarwal et al., "Distributions of charged hadrons associated with high transverse momentum particles in $p p$ and $\mathrm{Au}+\mathrm{Au}$ collisions at $\sqrt{s_{N N}}=200 \mathrm{GeV}$," Physical Review Letters, vol. 95, no. 15, Article ID 152301, 2005.

[44] A. Adare, S. S. Adler, S. Afanasiev et al., "System size and energy dependence of jet-induced hadron pair correlation shapes in $\mathrm{Cu}+\mathrm{Cu}$ and $\mathrm{Au}+\mathrm{Au}$ Collisions at $\sqrt{s_{N N}}=200$ and $62.4 \mathrm{GeV}$," Physical Review Letters, vol. 98, no. 23, Article ID 232302, 2007.

[45] B. Alver, B. B. Back, M. D. Baker et al., "System size dependence of cluster properties from two-particle angular correlations in $\mathrm{Cu}+\mathrm{Cu}$ and $\mathrm{Au}+\mathrm{Au}$ collisions at $\sqrt{s_{\mathrm{NN}}}=200 \mathrm{GeV}$," Physical Review C, vol. 81, no. 2, Article ID 024904, 9 pages, 2010.

[46] S. Chatrchyan, V. Khachatryan, A. M. Sirunyan et al., "Centrality dependence of dihadron correlations and azimuthal anisotropy harmonics in $\mathrm{PbPb}$ collisions at $\sqrt{s_{N N}}=2.76 \mathrm{TeV}$," The European Physical Journal C, vol. 72, article 2012, 2012.

[47] A. Dumitru, F. Gelis, L. McLerran, and R. Venugopalan, "Glasma flux tubes and the near side ridge phenomenon at RHIC," Nuclear Physics A, vol. 810, no. 1-4, pp. 91-108, 2008.

[48] CMS Collaboration, "Observation of particle-species dependence and collectivity in azimuthal correlations of pp collisions at the LHC", http://inspirehep.net/record/1465558?ln=en.

[49] I. Nakagawa, (PHENIX), Quark Matter presentations.

[50] K. Dusling, W. Li, and B. Schenke, "Novel collective phenomena in high-energy proton-proton and proton-nucleus collisions," International Journal of Modern Physics E: Nuclear Physics, vol. 25, no. 1, Article ID 1630002, 77 pages, 2016.

[51] C. Loizides, "Experimental overview on small collision systems at the LHC," https://arxiv.org/abs/1602.09138.
[52] S. Chatrchyan, V. Khachatryan, A. M. Sirunyan et al., "Observation and studies of jet quenching in $\mathrm{PbPb}$ collisions at $\sqrt{s_{N N}}=$ 2.76 TeV," Physical Review C, vol. 84, Article ID 024906, 2011.

[53] C. Adler, Z. Ahammed, C. Allgower et al., "Disappearance of back-to-back high-pT hadron correlations in central $\mathrm{Au}+\mathrm{Au}$ collisions at $\sqrt{s_{\mathrm{NN}}}=200 \mathrm{GeV}$," Physical Review Letters, vol. 90, no. 8, Article ID 082302, 6 pages, 2003.

[54] G. Aad, B. Abbott, J. Abdallah et al., "Centrality and rapidity dependence of inclusive jet production in $\sqrt{s_{N N}}=5.02 \mathrm{TeV}$ proton-lead collisions with the ATLAS detector," Physics Letters $B$, vol. 748, pp. 392-413, 2015.

[55] J. Adam, D. Adamová, M. M. Aggarwal et al., "Centrality dependence of particle production in $p$ - $\mathrm{Pb}$ collisions at $\sqrt{{s_{N N}}_{2}}=$ 5.02 TeV," Physical Review C, vol. 91, Article ID 064905, 2015.

[56] A. Adare, C. Aidala, N. N. Ajitanand et al., "Centralitydependent modification of jet-production rates in deuterongold collisions at $\sqrt{s_{\mathrm{NN}}}=200 \mathrm{GeV}$," Physical Review Letters, vol. 116, no. 12, Article ID 122301, 9 pages, 2016.

[57] J. Adam, D. Adamova, M. M. Aggarwal et al., "Centrality dependence of charged jet production in $\mathrm{p}-\mathrm{Pb}$ collisions at $\sqrt{s_{N N}}=5.02 \mathrm{TeV}$,' The European Physical Journal C, vol. 76, article 271, 2016.

[58] L. He, T. Edmonds, Z.-W. Lin, F. Liu, D. Molnar, and F. Wang, "Anisotropic parton escape is the dominant source of azimuthal anisotropy in transport models," Physics Letters B, vol. 753, pp. 506-510, 2016.

[59] S. Schlichting, "Initial state and pre-equilibrium effects in small systems," https://arxiv.org/abs/1601.01177.

[60] H. Mäntysaari and B. Schenke, "Evidence of strong proton shape fluctuations from incoherent diffraction," Physical Review Letters, vol. 117, no. 5, Article ID 052301, 7 pages, 2016.

[61] L. McLerran and P. Tribedy, "Intrinsic fluctuations of the proton saturation momentum scale in high multiplicity $\mathrm{p}+\mathrm{p}$ collisions," Nuclear Physics A, vol. 945, pp. 216-225, 2016.

[62] P. Tribedy, "Multi-particle production in small colliding systems from the Color Glass Condensate," in Proceedings of the 7th International Workshop on Multiple Partonic Interactions at the LHC (MPI@LHC '15), pp. 224-227, November 2015.

[63] G. Aad, B. Abbott, J. Abdallah et al., "Measurement of longrange pseudorapidity correlations and azimuthal harmonics in $\sqrt{s_{N N}}=5.02 \mathrm{TeV}$ proton-lead collisions with the ATLAS detector," Physical Review C, vol. 90, Article ID 044906, 2014.

[64] S. Chatrchyan, V. Khachatryan, A. M. Sirunyan et al., "Multiplicity and transverse momentum dependence of two- and fourparticle correlations in $\mathrm{pPb}$ and $\mathrm{PbPb}$ collisions," Physics Letters $B$, vol. 724, no. 4-5, pp. 213-240, 2013.

[65] D. Teaney, Initial stages 2016 presentation, 2016.

[66] A. Kurkela and Y. Zhu, "Isotropization and hydrodynamization in weakly coupled heavy-ion collisions," Physical Review Letters, vol. 115, no. 18, Article ID 182301, 5 pages, 2015.

[67] L. Keegan, A. Kurkela, A. Mazeliauskas, and D. Teaney, "Initial conditions for hydrodynamics from weakly coupled preequilibrium evolution," Journal of High Energy Physics, vol. 2016, article 171, 2016.

[68] T. Lappi, "Energy dependence of the saturation scale and the charged multiplicity in pp and AA collisions," The European Physical Journal C, vol. 71, article 1699, 2011.

[69] J. Adam and ALICE Collaboration, "Charged-particle multiplicities in proton-proton collisions at $\sqrt{s}=0.9$ to $8 \mathrm{TeV}$," https://arxiv.org/abs/1509.07541. 
[70] B. Abelev, J. Adam, Adamová et al., "Pseudorapidity density of charged particles in $p+\mathrm{Pb}$ collisions at $\sqrt{s_{\mathrm{NN}}}=5.02 \mathrm{TeV}$," Physical Review Letters, vol. 110, no. 3, Article ID 032301, 10 pages, 2013.

[71] D. d'Enterria, G. K. Eyyubova, V. L. Korotkikh et al., "Estimates of hadron azimuthal anisotropy from multiparton interactions in proton-proton collisions at $\sqrt{s}=14 \mathrm{TeV}$,' The European Physical Journal C, vol. 66, no. 1-2, pp. 173-185, 2010.

[72] B. Schenke, P. Tribedy, and R. Venugopalan, "Fluctuating glasma initial conditions and flow in heavy ion collisions," Physical Review Letters, vol. 108, no. 25, Article ID 252301, 2012.

[73] B. Schenke, P. Tribedy, and R. Venugopalan, "Event-by-event gluon multiplicity, energy density, and eccentricities in ultrarelativistic heavy-ion collisions," Physical Review C, vol. 86, no. 3, Article ID 034908, 2012.

[74] E. Iancu, A. H. Mueller, and S. Munier, "Universal behavior of QCD amplitudes at high energy from general tools of statistical physics," Physics Letters B, vol. 606, no. 3-4, pp. 342-350, 2005.

[75] E. Iancu and D. N. Triantafyllopoulos, "A Langevin equation for high-energy evolution with pomeron loops," Nuclear Physics A, vol. 756, no. 3-4, pp. 419-467, 2005.

[76] S. Munier and R. B. Peschanski, "Traveling wave fronts and the transition to saturation," Physical Review D, vol. 69, Article ID 034008, 2004.

[77] S. Munier and R. Peschanski, "Geometric scaling as traveling waves," Physical Review Letters, vol. 91, no. 23, Article ID 232001, 3 pages, 2003.

[78] A. H. Mueller and S. Munier, "On parton number fluctuations at various stages of the rapidity evolution," Physics Letters B, vol. 737, pp. 303-310, 2014.

[79] C. Marquet, G. Soyez, and B.-W. Xiao, "On the probability distribution of the stochastic saturation scale in QCD," Physics Letters B, vol. 639, no. 6, pp. 635-641, 2006.

[80] A. Bzdak and K. Dusling, "Probing proton fluctuations with asymmetric rapidity correlations," Physical Review C, vol. 93, no. 3, Article ID 031901, 2016.

[81] H. Kowalski, L. Motyka, and G. Watt, "Exclusive diffractive processes at HERA within the dipole picture," Physical Review D, vol. 74, no. 7, Article ID 074016, 27 pages, 2006.

[82] A. H. Rezaeian, M. Siddikov, M. Van de Klundert, and R. Venugopalan, "Analysis of combined HERA data in the impactparameter dependent saturation model," Physical Review D, vol. 87, Article ID 034002, 2013.

[83] S. Schlichting and B. Schenke, "The shape of the proton at high energies," Physics Letters B, vol. 739, pp. 313-319, 2014.

[84] K. Welsh, J. Singer, and U. W. Heinz, "Initial state fluctuations in collisions between light and heavy ions," Physical Review C, vol. 94, no. 2, Article ID 024919, 2016.

[85] P. Bozek, W. Broniowski, and M. Rybczynski, "Wounded quarks in $A+A, p+A$, and $p+p$ collisions," Physical Review $C$, vol. 94, Article ID 014902, 2016.

[86] J. L. Albacete and A. Soto-Ontoso, "Hot spots and the hollowness of proton-proton interactions at high energies," https://arxiv.org/abs/1605.09176.

[87] F. Gelis, T. Lappi, and L. McLerran, “Glittering glasmas," Nuclear Physics A, vol. 828, no. 1-2, pp. 149-160, 2009.

[88] A. H. Mueller and H. Navelet, "An inclusive minijet cross section and the bare pomeron in QCD," Nuclear Physics B, vol. 282, pp. 727-744, 1987.

[89] J. P. Blaizot, F. Gelis, and R. Venugopalan, "High energy $p A$ collisions in the color glass condensate approach I: gluon production and the Cronin effect," Nuclear Physics A, vol. 743, no. 1-3, pp. 13-56, 2004.

[90] I. Balitsky, "Operator expansion for high-energy scattering," Nuclear Physics B, vol. 463, no. 1, pp. 99-157, 1996.

[91] Y. V. Kovchegov, "Small- $x F_{2}$ structure function of a nucleus including multiple Pomeron exchanges," Physical Review D, vol. 60, Article ID 034008, 1999.

[92] Y. V. Kovchegov, "Unitarization of the BFKL Pomeron on a nucleus," Physical Review D, vol. 61, no. 7, Article ID 074018, 15 pages, 2000.

[93] J. Jalilian-Marian, A. Kovner, L. McLerran, and H. Weigert, "Intrinsic glue distribution at very small x," Physical Review DParticles, Fields, Gravitation and Cosmology, vol. 55, no. 9, pp. 5414-5428, 1997.

[94] E. Iancu, A. Leonidov, and L. D. McLerran, "Nonlinear gluon evolution in the color glass condensate: I," Nuclear Physics A, vol. 692, no. 3-4, pp. 583-645, 2001.

[95] E. Ferreiro, E. Iancu, A. Leonidov, and L. McLerran, "Nonlinear gluon evolution in the color glass condensate: II," Nuclear Physics A, vol. 703, no. 1-2, pp. 489-538, 2002.

[96] I. Balitsky, "Quark contribution to the small- $x$ evolution of color dipole," Physical Review D, vol. 75, no. 1, Article ID 014001, 21 pages, 2007.

[97] L. D. McLerran and R. Venugopalan, "Computing quark and gluon distribution functions for very large nuclei," Physical Review D, vol. 49, no. 5, Article ID 2233, 1994.

[98] L. D. McLerran and R. Venugopalan, "Gluon distribution functions for very large nuclei at small transverse momentum," Physical Review D, vol. 49, no. 7, p. 3352, 1994.

[99] J. L. Albacete, N. Armesto, J. G. Milhano, P. Q. Arias, and C. A. Salgado, "AAMQS: a non-linear QCD analysis of new HERA data at small- $x$ including heavy quarks," The European Physical Journal C, vol. 71, no. 7, pp. 1-11, 2011.

[100] H. Kowalski and D. Teaney, "Impact parameter dipole saturation model," Physical Review D, vol. 68, Article ID 114005, 2003.

[101] E. Iancu, J. D. Madrigal, A. H. Mueller, G. Soyez, and D. N. Triantafyllopoulos, "Resumming double logarithms in the QCD evolution of color dipoles," Physics Letters B, vol. 744, pp. 293-302, 2015.

[102] E. Iancu, J. D. Madrigal, A. H. Mueller, G. Soyez, and D. N. Triantafyllopoulos, "Collinearly-improved BK evolution meets the HERA data," Physics Letters B, vol. 750, pp. 643-652, 2015.

[103] T. Lappi and H. Mäntysaari, "Next-to-leading order BalitskyKovchegov equation with resummation," Physical Review D, vol. 93, Article ID 094004, 2016.

[104] D. Colferai, F. Schwennsen, L. Szymanowski, and S. Wallon, "Mueller Navelet jets at LHC_complete next-to-leading BFKL calculation," Journal of High Energy Physics, vol. 2010, no. 12, article 26, 2010.

[105] V. S. Fadin, M. Kotsky, and L. Lipatov, "Gluon pair production in the quasi-multi-regge kinematics," https://arxiv.org/abs/ hep-ph/9704267.

[106] A. Leonidov and D. Ostrovsky, "Angular and momentum asymmetry in particle production at high energies," Physical Review D, vol. 62, no. 9, Article ID 094009, 2000.

[107] T. Lappi, S. Srednyak, and R. Venugopalan, "Non-perturbative computation of double inclusive gluon production in the Glasma," Journal of High Energy Physics, vol. 2010, no. 1, article 66,2010

[108] P. Tribedy and R. Venugopalan, "QCD saturation at the LHC: comparisons of models to $\mathrm{p}+\mathrm{p}$ and $\mathrm{A}+\mathrm{A}$ data and predictions 
for $\mathrm{p}+\mathrm{Pb}$ collisions," Physics Letters B, vol. 710, no. 1, pp. 125133, 2012, Erratum: Physics Letters B, vol. 718, no. 3, pp. 1154, 2013.

[109] P. Tribedy and R. Venugopalan, "Saturation models of HERA DIS data and inclusive hadron distributions in $\mathrm{p}+\mathrm{p}$ collisions at the LHC," Nuclear Physics A, vol. 850, no. 1, pp. 136-156, 2011, Erratum in: Nuclear Physics A, vol. 859, no. 1, pp. 185-187, 2011.

[110] T. Altinoluk, N. Armesto, G. Beuf, A. Kovner, and M. Lublinsky, "Bose enhancement and the ridge," Physics Letters, Section B: Nuclear, Elementary Particle and High-Energy Physics, vol. 751, pp. 448-452, 2015.

[111] E. Iancu and D. N. Triantafyllopoulos, "JIMWLK evolution for multi-particle production in Langevin form," Journal of High Energy Physics, vol. 2013, article 67, 2013.

[112] A. Dumitru, A. V. Giannini, and V. Skokov, "Anisotropic particle production and azimuthal correlations in high-energy pA collisions," https://arxiv.org/abs/1503.03897.

[113] L. McLerran and V. Skokov, "Finite numbers of sources, particle correlations and the Color Glass Condensate," Nuclear Physics A, vol. 947, pp. 142-154, 2016.

[114] L. McLerran and R. Venugopalan, "Green's function in the color field of a large nucleus," Physical Review D, vol. 50, no. 3, pp. 2225-2233, 1994.

[115] L. McLerran and R. Venugopalan, "Gluon distribution functions for very large nuclei at small transverse momentum," Physical Review D, vol. 49, no. 7, pp. 3352-3355, 1994.

[116] J. Jalilian-Marian, A. Kovner, L. D. McLerran, and H. Weigert, "Intrinsic glue distribution at very small $x$," Physical Review D, vol. 55, no. 9, pp. 5414-5428, 1997.

[117] Y. V. Kovchegov, "Non-Abelian Weizsäcker-Williams field and a two-dimensional effective color charge density for a very large nucleus," Physical Review D, vol. 54, no. 9, pp. 5463-5469, 1996.

[118] A. Kovner, L. D. McLerran, and H. Weigert, "Gluon production from non-Abelian Weizsäcker-Williams fields in nucleusnucleus collisions," Physical Review D, vol. 52, pp. 6231-6237, 1995.

[119] A. Kovner, L. D. McLerran, and H. Weigert, "Gluon production at high transverse momentum in the McLerran-Venugopalan model of nuclear structure functions," Physical Review D, vol. 52, no. 7, p. 3809, 1995.

[120] J. Berges, K. Boguslavski, S. Schlichting, and R. Venugopalan, "Universal attractor in a highly occupied non-Abelian plasma," Physical Review D, vol. 89, no. 11, Article ID 114007, 45 pages, 2014.

[121] J. Berges, K. Boguslavski, S. Schlichting, and R. Venugopalan, "Turbulent thermalization process in heavy-ion collisions at ultrarelativistic energies," Physical Review D, vol. 89, Article ID 074011, 2014.

[122] A. Adare, C. Aidala, N. N. Ajitanand et al., "Quadrupole anisotropy in dihadron azimuthal correlations in central $d+\mathrm{Au}$ Collisions at $\sqrt{s_{N N}}=200 \mathrm{GeV}$,' Physical Review Letters, vol. 111, no. 21, Article ID 212301, 2013.

[123] R. D. Field and R. P. Feynman, "A parametrization of the properties of quark jets," Nuclear Physics B, vol. 136, no. 1, pp. 1-76, 1978.

[124] B. A. Kniehl, G. Kramer, and B. Potter, "Fragmentation functions for pions, kaons, and protons at next-to-leading order," Nuclear Physics B, vol. 582, no. 1-3, pp. 514-536, 2000.

[125] D. de Florian, R. Sassot, and M. Stratmann, "Global analysis of fragmentation functions for pions and kaons and their uncertainties," Physical Review D, vol. 75, no. 11, Article ID 114010, 26 pages, 2007.
[126] Y. I. Azimov, Y. L. Dokshitzer, V. A. Khoze, and S. I. Troyan, “The string effect and QCD coherence," Physics Letters B, vol. 165, no. 1-3, pp. 147-150, 1985.

[127] E. Levin and A. H. Rezaeian, "Gluon saturation and inclusive hadron production at LHC," Physical Review D, vol. 82, no. 1, Article ID 014022, 2010.

[128] B. Andersson, G. Gustafson, G. Ingelman, and T. Sjöstrand, "Parton fragmentation and string dynamics," Physics Reports, vol. 97, no. 2-3, pp. 31-145, 1983.

[129] T. Sjostrand, "High-energy-physics event generation with PYTHIA 5.7 and JETSET 7.4," Computer Physics Communications, vol. 82, no. 1, pp. 74-89, 1994.

[130] A. Esposito and M. Gyulassy, "Hadronization scheme dependence of long-range azimuthal harmonics in high energy $\mathrm{p}+\mathrm{A}$ reactions," Physics Letters B, vol. 747, pp. 433-440, 2015.

[131] A. Ortiz Velasquez, P. Christiansen, E. Cuautle Flores, I. A. Maldonado Cervantes, and G. Paić, "Color reconnection and flowlike patterns in pp collisions," Physical Review Letters, vol. 111, no. 4, Article ID 042001, 2013.

[132] Y. Zhou, X. Zhu, P. Li, and H. Song, "Investigation of possible hadronic flow in $\sqrt{s_{N N}}=5.02 \mathrm{TeV} p-\mathrm{Pb}$ collisions," Physical Review C, vol. 91, no. 6, Article ID 064908, 2015.

[133] B. Schenke, S. Schlichting, P. Tribedy, and R. Venugopalan, "Mass ordering of spectra from fragmentation of saturated gluon states in high multiplicity proton-proton collisions," https://arxiv.org/abs/1607.02496.

[134] H. Niemi and G. S. Denicol, "How large is the Knudsen number reached in fluid dynamical simulations of ultrarelativistic heavy ion collisions?" https://arxiv.org/abs/1404.7327.

[135] B. B. Abelev, J. Adamaj, D. Adamová et al., "Long-range angular correlations of $\pi, \mathrm{K}$ and $\mathrm{p}$ in $\mathrm{p}-\mathrm{Pb}$ collisions at $\sqrt{s_{N N}}=$ 5.02 TeV," Physics Letters B, vol. 726, no. 1-3, pp. 164-177, 2013.

[136] V. Khachatryan, A. M. Sirunyan, A. Tumasyan et al., "Evidence for collective multiparticle correlations in $p-\mathrm{Pb}$ collisions," Physical Review Letters, vol. 115, no. 1, Article ID 012301, 17 pages, 2015.

[137] G. Aad and ATLAS Collaboration, "Multiparticle azimuthal correlations in $p-\mathrm{Pb}$ and $\mathrm{Pb}-\mathrm{Pb}$ collisions at the CERN large hadron collider," Physical Review C, vol. 90, no. 5, Article ID 054901, 2014

[138] V. Skokov, "High order cumulants of the azimuthal anisotropy in the dilute-dense limit: connected graphs," Physical Review D, vol. 91, Article ID 054014, 2015.

[139] S. Schlichting, "Initial State and Pre-Equilibrium Effects in Small Systems," Quark Matter presentations, 2015, https://indico.cern.ch/event/355454/contributions/838331/attachments/ 1163825/1676823/SchlichtingQM2015.pdf. 

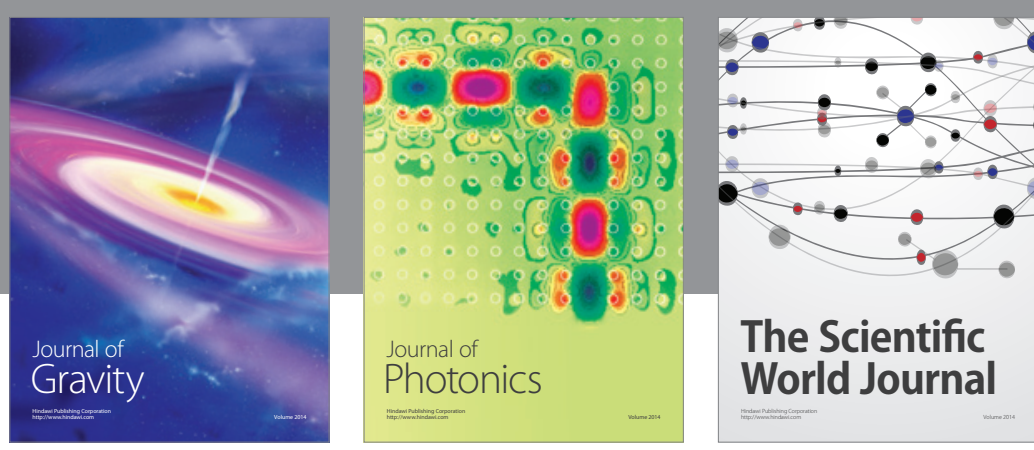

The Scientific World Journal
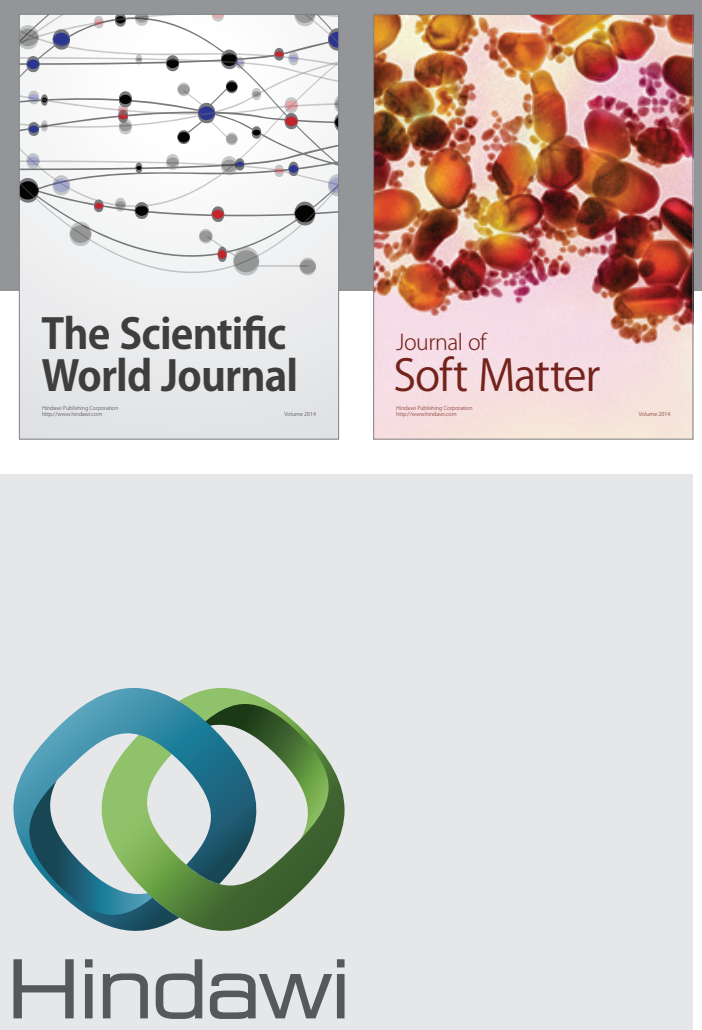

Submit your manuscripts at

http://www.hindawi.com

nternational Journal of

Statistical Mechanics
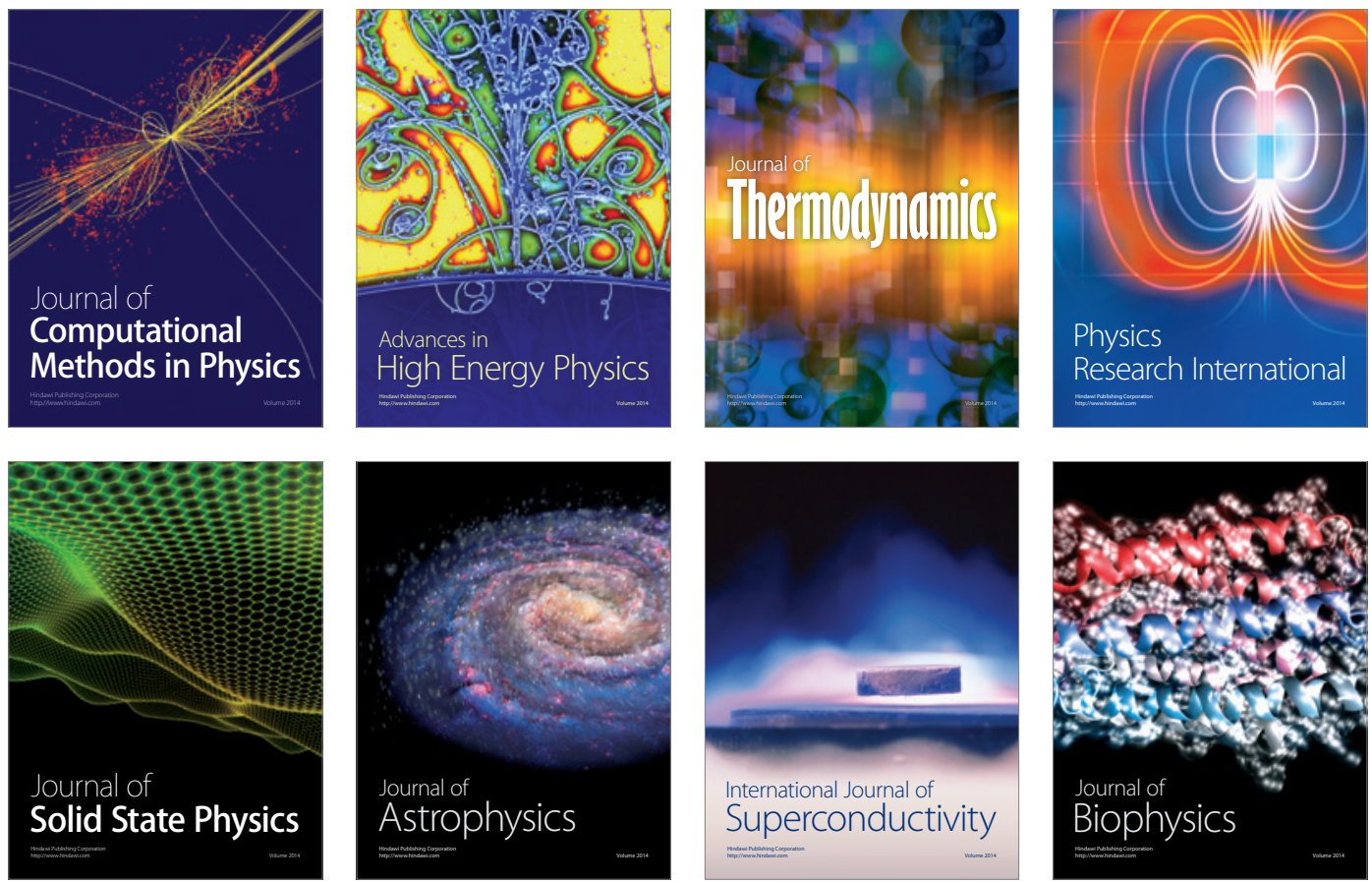
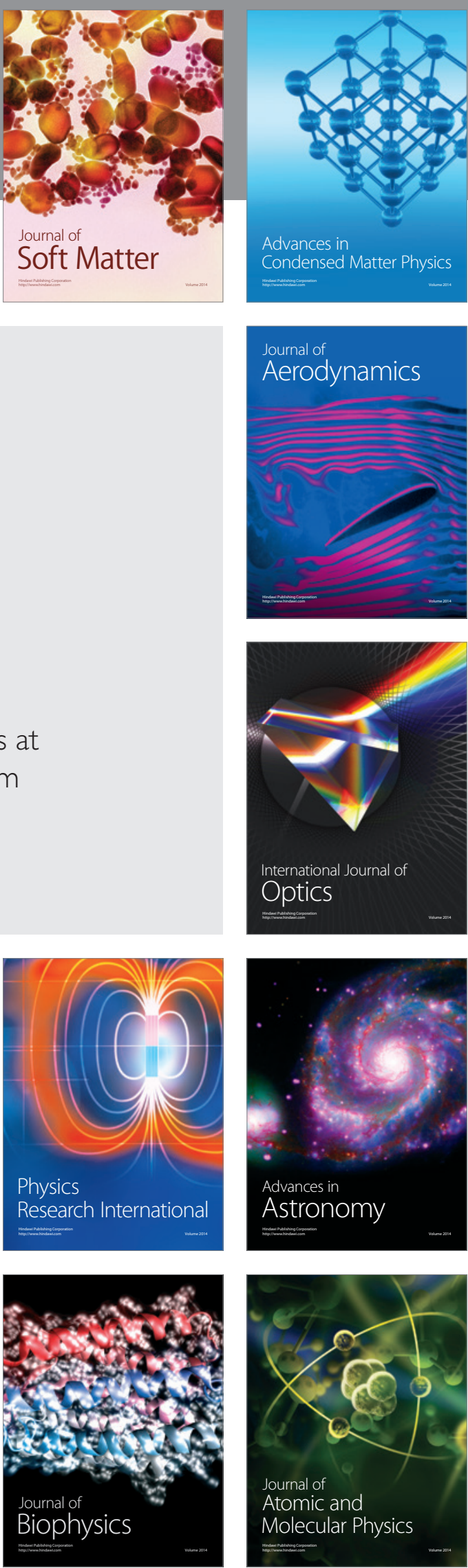